\title{
। \\ CORRELATION OF SHIFTED VALUES OF L-FUNCTIONS IN THE HYPERELLIPTIC ENSEMBLE
}

\author{
PRANENDU DARBAR AND GOPAL MAITI
}

\begin{abstract}
The moments of quadratic Dirichlet $L$-functions over function fields have recently attracted much attention with the work of Andrade and Keating. In this article, we establish lower bounds for the mean values of the product of quadratic Dirichlet $L$ functions associated with hyperelliptic curves of genus $g$ over a fixed finite field $\mathbb{F}_{q}$ in the large genus limit. By using the idea of A. Florea [14, we also obtain their upper bounds. As a consequence, we find upper bounds of its derivatives. These lower and upper bounds give the correlation of quadratic Dirichlet $L$-functions associated with hyperelliptic curves with different transitions.
\end{abstract}

\section{INTRODUCTION}

The correlation of $L$-functions i.e., study of the mean values of product of shifted values of $L$-functions near the critical line has become central to number theory. Random matrix theory has recently become a fundamental tool for understanding the correlation of $L$-functions. Montgomery [22] showed that two-point correlations between the non-trivial zeros of the Riemann $\zeta$-function, on the scale of the mean zero spacing, are similar to the corresponding correlations between the eigenvalues of random unitary matrices in the limit of large matrix size and conjectured that these correlations are, in fact, identical to each other.

Keating and Snaith [21] suggested that the value distribution of the Riemann zeta function on its critical line is related to that the characteristic polynomials of random unitary matrices. Conjectures for the moments of $L$-functions have been attempted for many decades, with very little progress until the random matrix theory came into the subject.

The main observation is that the structure of the mean values of L-functions is more clearly revealed if one considers the average of a product of $L$-functions, where each $L$-function is evaluated at a location slightly shifted from the critical point.

In this article, we discus about the moments and correlation of Riemann zeta function (belonging to unitary family), Dirichlet $L$-functions (contained in the symplectic family) and quadratic Dirichlet $L$-functions associated with hyperelliptic curves of large genus over a fixed finite field which are also members of the symplectic family. These families and their random matrix analogs have been discussed from the perspective of the leading terms in the asymptotic expressions by several authors (see [8], [9], [10], 3], [20], 21], [11] and [19]).

Our main goal of this article is to establish lower and upper bounds for the correlation of shifted values of quadratic Dirichlet $L$-functions near the critical line associated to the hyperelliptic curves of large genus over a fixed finite field.

2010 Mathematics Subject Classification: 11G20,11T06, 11M50.

Key words and phrases. Finite fields, Function fields, Correlations of quadratic Dirichlet $L$-functions, Hyperelliptic curves. 
1.1. Moments of the Riemann Zeta function. A classical question in the theory of Riemann zeta function is to determine the asymptotic behaviour of

$$
M_{k}(T):=\int_{1}^{T}\left|\zeta\left(\frac{1}{2}+i t\right)\right|^{2 k} d t
$$

where $k \in \mathbb{C}$, as $T \rightarrow \infty$. It is believed that for a given positive real number $k$,

$$
M_{k}(T) \sim c_{k} T(\log T)^{k^{2}}
$$

where $c_{k}$ is a positive constant. Ramachandra [27] showed that

$$
M_{k}(T) \gg T(\log T)^{k^{2}}
$$

for any $k \in \mathbb{N}$. Using moments of characteristic polynomials of random matrices, Keating and Snaith [20], conjectured an exact value of $c_{k}$ for $\Re(k)>-\frac{1}{2}$. Assuming the Riemann hypothesis $(\mathrm{RH})$, Soundararajan [33] showed that for every positive real number $k$ and $\varepsilon>0$,

$$
M_{k}(T) \ll_{k, \varepsilon} c_{k} T(\log T)^{k^{2}+\varepsilon}
$$

and Harper [17] removed the exponent $\epsilon$ in the bound of (1.1).

A generalization of the moments of $\zeta(s)$ are the shifted moments, defined as

$$
M_{\boldsymbol{k}^{(m)}}\left(T, \boldsymbol{\alpha}^{(m)}\right):=\int_{0}^{T}\left|\zeta\left(\frac{1}{2}+i t+i \alpha_{1}\right)\right|^{2 k_{1}} \ldots\left|\zeta\left(\frac{1}{2}+i t+i \alpha_{m}\right)\right|^{2 k_{m}} d t
$$

where $\boldsymbol{k}^{(m)}=\left(k_{1}, \ldots, k_{m}\right)$ is a sequence of positive real numbers and $\boldsymbol{\alpha}^{(m)}=\left(\alpha_{1}, \ldots, \alpha_{m}\right) \in$ $\mathbb{R}^{m}$ with $\alpha_{i} \neq \alpha_{j}$ for $i \neq j$. In [7], Chandee obtained lower and upper bounds of $M_{\boldsymbol{k}^{(m)}}\left(T, \boldsymbol{\alpha}^{(m)}\right)$ for some special choices of $\boldsymbol{\alpha}^{(m)}$ and for a large values of $T$. More precisely, she proved assuming the RH,

$$
M_{\boldsymbol{k}^{(m)}}\left(T, \boldsymbol{\alpha}^{(m)}\right) \ll_{\boldsymbol{k}^{(m)}, \varepsilon} T(\log T)^{k_{1}^{2}+\ldots+k_{m}^{2}+\varepsilon} \prod_{i<j}\left(\min \left\{\frac{1}{\left|\alpha_{i}-\alpha_{j}\right|}, \log T\right\}\right)^{2 k_{i} k_{j}}
$$

and unconditionally

$$
M_{\boldsymbol{k}^{(m)}}\left(T, \boldsymbol{\alpha}^{(m)}\right) \gg_{\boldsymbol{k}^{(m)}, \boldsymbol{\alpha}^{(m)}} T(\log T)^{k_{1}^{2}+\ldots+k_{m}^{2}} \prod_{i<j}\left(\min \left\{\frac{1}{\left|\alpha_{i}-\alpha_{j}\right|}, \log T\right\}\right)^{2 k_{i} k_{j}}
$$

for sufficiently large $T$.

The moments of the derivatives of the Riemann zeta function were studied by several mathematicians. An analog of Soundararajan's estimate (1.1) for the derivatives of Riemann zeta function was obtained by Milinovich [24]. Under the RH, he showed that for every $\varepsilon>0$,

$$
\int_{1}^{T}\left|\zeta^{(l)}\left(\frac{1}{2}+i t\right)\right|^{2 k} d t \ll_{k, l, \varepsilon} T(\log T)^{k^{2}+2 k l+\varepsilon},
$$

where $k, l \in \mathbb{N}$ and $\zeta^{(l)}$ is the $l$-th derivative of $\zeta$. 
1.2. Moments of quadratic Dirichlet $L$-functions. Let $\chi_{d}$ be a real primitive Dirichlet character modulo $d$ given by the Kronecker symbol $\chi_{d}(n)=\left(\frac{d}{n}\right)$. It is interesting to determine the asymptotic behaviour of $\sum_{0<d \leq D} L\left(\frac{1}{2}, \chi_{d}\right)^{k}$ as $D \rightarrow \infty$. Extending their approach to the zeta function, using random matrix theory, Keating and Snaith [21] made the following conjecture about the asymptotic behaviour of moments of Dirichlet $L$-functions $L\left(\frac{1}{2}, \chi_{d}\right)$.

Conjecture (Keating, Snaith). For $k$ fixed with $\Re(k) \geq 0$, as $D \rightarrow \infty$

$$
\frac{1}{D} \sum_{|d| \leq D}^{\star} L\left(\frac{1}{2}, \chi_{d}\right)^{k} \sim c_{k}(\log D)^{\frac{k(k+1)}{2}}
$$

where $c_{k}$ is a positive constant and $\sum^{\star}$ indicates that the sum is over fundamental discriminants.

In [30], Rudnick and Soundararajan obtained that for any rational number $k \geq 1$,

$$
\frac{1}{D} \sum_{|d| \leq D}^{\star} L\left(\frac{1}{2}, \chi_{d}\right)^{k} \gg_{k}(\log D)^{\frac{k(k+1)}{2}} .
$$

Assuming the generalized Riemann hypothesis (GRH), Soundararajan established that for any positive real number $k$ and $\epsilon>0$,

$$
\frac{1}{D} \sum_{|d| \leq D}^{\star} L\left(\frac{1}{2}, \chi_{d}\right)^{k} \ll_{k, \epsilon}(\log D)^{\frac{k(k+1)}{2}+\varepsilon} .
$$

In general, it is important to find asymptotic behaviour of the following correlation of shifted values of Dirichlet $L$-functions:

$$
S_{\boldsymbol{k}^{(m)}}\left(\boldsymbol{\alpha}^{(m)}, D\right):=\sum_{d \leq D}^{\star} L\left(\frac{1}{2}+\alpha_{1}, \chi_{d}\right)^{k_{1}} \ldots L\left(\frac{1}{2}+\alpha_{m}, \chi_{d}\right)^{k_{m}},
$$

where $\boldsymbol{k}^{(m)}=\left(k_{1}, \ldots, k_{m}\right)$ be a sequence of real numbers and $\boldsymbol{\alpha}^{(m)}=\left(\alpha_{1}, \ldots, \alpha_{m}\right)$ be a sequence of complex numbers with $\alpha_{i} \neq \alpha_{j}$ for $i \neq j$.

Conrey et. al. [9] gave a conjecture on the asymptotic behaviour of $S_{\boldsymbol{k}^{(m)}}\left(\boldsymbol{\alpha}^{(m)}, D\right)$. Analogous questions for higher degree $L$-functions have been studied by Milinovich and TurnageButterbaugh [26].

1.3. Moments of $L$-functions in the hyperelliptic essemble. Let $\mathbb{F}_{q}$ be a finite field of odd cardinality and $\mathbb{F}_{q}[t]$ be the polynomial ring over $\mathbb{F}_{q}$ in variable $t$. Let $D \in \mathbb{F}_{q}[t]$ be a monic square-free polynomial. The quadratic character $\chi_{D}$ attached to $D$ is defined using quadratic residue symbol for $\mathbb{F}_{q}[t]$ by $\chi_{D}(f)=\left(\frac{D}{f}\right)$ and the corresponding Dirichlet $L$ function is denoted by $L\left(s, \chi_{D}\right)$. It is often convenient to work with the equivalent $L$-function $\mathcal{L}\left(u, \chi_{D}\right)$ written in terms of the variable $u=q^{-s}$.

Define hyperelltiptic ensemble $\mathcal{H}_{n, q}$ or simply $\mathcal{H}_{n}$ as

$$
\mathcal{H}_{n}=\left\{D \in \mathbb{F}_{q}[t]: D \text { is monic, square free, and } \operatorname{deg}(D)=n\right\} .
$$

For each $D$ in the Hyperelliptic essemble $\mathcal{H}_{n}$, there is an associated hyperelliptic curve given by $C_{D}: y^{2}=D(t)$. These curve are non-singular and of genus $g$ given by

$$
2 g=n-1-\lambda,
$$


where

$$
\lambda= \begin{cases}1, & \text { if } n \text { even } \\ 0, & \text { if } n \text { odd }\end{cases}
$$

Note that, $g \rightarrow \infty$ as $n$ does so. See section 2 for more details about the properties of Dirichlet $L$-function $L\left(s, \chi_{D}\right)$ and their spectral interpretation.

Andrade and Keating [3] conjectured that as $g \rightarrow \infty$,

$$
\sum_{D \in \mathcal{H}_{2 g+1}} L\left(\frac{1}{2}, \chi_{D}\right)^{k}=q^{2 g+1}\left(P_{k}(2 g+1)+o(1)\right)
$$

where $P_{k}$ is a polynomial of degree $\frac{k(k+1)}{2}$. Assuming $q \equiv 1(\bmod 4)$, the conjecture (1.3) is known for $k=1$ from the work of Andrade [2], and the error term in the asymptotic formula was improved by Florea [12]. In [13, 14, Florea also proved the conjecture (1.3) for $k=2,3$ and 4 assuming $q \equiv 1(\bmod 4)$. For $n=2 g+2$, Jung [18] obtained that

$$
\frac{1}{\left|\mathcal{H}_{2 g+2}\right|} \sum_{D \in \mathcal{H}_{2 g+2}} L\left(\frac{1}{2}, \chi_{D}\right)=P(1)(g+1)+\frac{P^{\prime}(1)}{\log q}-P(1) \zeta_{\mathbb{A}}\left(\frac{1}{2}\right)+O\left(2^{g+1} q^{-\frac{g}{2}}\right),
$$

where $P(s)=\prod_{P}\left(1-(1+|P|)^{-1}|P|^{-s}\right)$ and $\zeta_{\mathbb{A}}\left(\frac{1}{2}\right)$ is defined in Section 2 .

Andrade [1] established the following lower bound:

Theorem 1.1 (Andrade). For every even natural number $k$, we have

$$
\frac{1}{\left|\mathcal{H}_{n}\right|} \sum_{D \in \mathcal{H}_{n}} L\left(\frac{1}{2}, \chi_{D}\right)^{k} \gg_{k} n^{\frac{k(k+1)}{2}}
$$

On the other hand, A. Florea [[14], Theorem 2.7] found the following upper bound for a single shifted $L$-function associated with hyperelliptic curves:

Theorem 1.2 (Florea). Let $v=e^{i \theta}$, with $\theta \in[0, \pi)$. Then for every positive $k$ and any $\epsilon>0$,

$$
\sum_{D \in \mathcal{H}_{2 g+1}}\left|\mathcal{L}\left(\frac{v}{\sqrt{q}}, \chi_{D}\right)\right|^{k} \ll_{k, \varepsilon} q^{2 g+1} g^{\varepsilon} \exp \left(k \mathcal{M}(v, g)+\frac{k^{2}}{2} \mathcal{V}(v, g)\right),
$$

where $\mathcal{M}(v, g)=\frac{1}{2} \log \left(\min \left\{g, \frac{1}{2 \theta}\right\}\right) \quad$ and $\quad \mathcal{V}(v, g)=\mathcal{M}(v, g)+\frac{1}{2} \log g$.

1.3.1. Shifted moments and main results. In this article, for fixed $m$-tuple $\boldsymbol{k}^{(m)}=\left(k_{1}, \ldots, k_{m}\right) \in$ $\mathbb{N}^{m}$, we shall investigate the following mean values of the product of $m$-shifted quadratic Dirichlet $L$-functions:

$$
\mathcal{S}_{n}\left(\boldsymbol{v}^{(m)}, \boldsymbol{k}^{(m)}\right):=\sum_{D \in \mathcal{H}_{n}} \mathcal{L}\left(\frac{v_{1}}{q^{\frac{1}{2}+\alpha_{1}}}, \chi_{D}\right)^{2 k_{1}} \ldots \mathcal{L}\left(\frac{v_{m}}{q^{\frac{1}{2}+\alpha_{m}}}, \chi_{D}\right)^{2 k_{m}}
$$

where $\boldsymbol{v}^{(m)}=\left(v_{1}, \ldots, v_{m}\right) \in \mathbb{C}^{m}$ with $v_{j}=e^{i \theta_{j}}, \theta_{j} \in(0, \pi]$ and $\alpha_{j} \in\left[0, \frac{1}{2}\right)$ for $j=1, \ldots, m$. Also, $\theta_{j}=\theta_{j}(g)$ is a real valued function of $g$ such that $\lim _{g \rightarrow \infty} g\left|\theta_{j}\right|$ and for $i \neq j, \lim _{g \rightarrow \infty} g\left|\theta_{i}-\theta_{j}\right|$ exists or equals $\infty$. Note that one can obtain the moments of $\mathcal{L}\left(\frac{v}{\sqrt{q}}, \chi_{D}\right)$ by allowing the shifts $\alpha_{j}$ to tend to 0 . 
Throughout the article, we follow that $n$ and $g$ are connected via (1.2). Before stating our main results, let us define

$$
\begin{aligned}
& \mu\left(\boldsymbol{v}^{(m)}, g\right)=\sum_{j=1}^{m} k_{j} \log \left(\min \left\{\frac{1}{2\left|\theta_{j}\right|}, g\right\}\right), \\
& \sigma\left(\boldsymbol{v}^{(m)}, g\right)=2\left(\sum_{j=1}^{m} k_{j}^{2}\right) \log g+2 \sum_{j=1}^{m} k_{j}^{2} \log \left(\min \left\{\frac{1}{2\left|\theta_{j}\right|}, g\right\}\right) \\
& +4 \sum_{i<j} k_{i} k_{j}\left(\log \left(\min \left\{\frac{1}{\left|\theta_{i}-\theta_{j}\right|}, g\right\}\right)+\log \left(\min \left\{\frac{1}{\left|\theta_{i}+\theta_{j}\right|}, g\right\}\right)\right) .
\end{aligned}
$$

For $m=2$, we set

$$
W=\left\{j \in\{1,2\}: \lim _{g \rightarrow \infty} g\left|\theta_{j}\right|<\infty\right\} \text { and } W^{c}=\left\{j \in\{1,2\}: \lim _{g \rightarrow \infty} g\left|\theta_{j}\right|=\infty\right\} .
$$

We also define a constant depending on $W$ and $W^{c}$ as

$$
c_{\boldsymbol{v}^{(2)}}=\max \left\{\lim _{g \rightarrow \infty} g\left|\theta_{1}\right|, \lim _{g \rightarrow \infty} g\left|\theta_{2}\right|, \lim _{g \rightarrow \infty} g\left|\theta_{1}-\theta_{2}\right|, \lim _{g \rightarrow \infty} g\left|\theta_{1}+\theta_{2}\right|\right\},
$$

where maximum is taken only over the finite entries of the set.

Remark 1. If $|W|=2$ and $\left|W^{c}\right|=0$ then

$$
c_{\boldsymbol{v}^{(2)}}=\max \left\{\lim _{g \rightarrow \infty} g\left|\theta_{1}\right|, \lim _{g \rightarrow \infty} g\left|\theta_{2}\right|, \lim _{g \rightarrow \infty} g\left|\theta_{1}-\theta_{2}\right|, \lim _{g \rightarrow \infty} g\left|\theta_{1}+\theta_{2}\right|\right\} .
$$

If $W=\{1\}$ and $W^{c}=\{2\}$ then $c_{\boldsymbol{v}^{(2)}}=\lim _{g \rightarrow \infty} g\left|\theta_{1}\right|$. If $W=\emptyset$ and $\lim _{g \rightarrow \infty} g\left|\theta_{1}-\theta_{2}\right|<\infty$, then $c_{\boldsymbol{v}^{(2)}}=\lim _{g \rightarrow \infty} g\left|\theta_{1}-\theta_{2}\right|$. When none of the limit is finite then $c_{\boldsymbol{v}^{(2)}}$ is an absolute constant.

We obtain a lower bound (of the conjectured order of magnitude $\rrbracket^{1}$ ) for $\mathcal{S}_{n}\left(\boldsymbol{v}^{(2)}, \boldsymbol{k}^{(2)}\right)$ in the large degree limit i.e. when $n$ is sufficiently large and $q$ is fixed.

Theorem 1.3. Let $\boldsymbol{k}^{(2)}$ and $\boldsymbol{v}^{(2)}$ be as earlier. Assume that $\alpha_{j}=O\left(\frac{1}{g}\right)$ for $j=1,2$. Then for $n$ large,

$$
\frac{1}{\left|\mathcal{H}_{n}\right|} \sum_{D \in \mathcal{H}_{n}}\left|\mathcal{L}\left(\frac{v_{1}}{q^{\frac{1}{2}+\alpha_{1}}}, \chi_{D}\right)\right|^{2 k_{1}}\left|\mathcal{L}\left(\frac{v_{2}}{q^{\frac{1}{2}+\alpha_{2}}}, \chi_{D}\right)\right|^{2 k_{2}} \gg_{\boldsymbol{k}^{(2)}, c_{\boldsymbol{v}}(2)} \exp \left(\mu\left(\boldsymbol{v}^{(2)}, g\right)+\frac{1}{2} \sigma\left(\boldsymbol{v}^{(2)}, g\right)\right),
$$

where $\mu\left(\boldsymbol{v}^{(2)}, g\right), \sigma\left(\boldsymbol{v}^{(2)}, g\right)$ and $c_{\boldsymbol{v}^{(2)}}$ are defined by (1.5), (1.6) and (1.8) respectively.

In this article, we will provide the complete proof of the Theorem 1.3 and from observations in the footnotes [2, 5, 7], one can easily extend Theorem 1.3 to the following form.

Theorem 1.4. Let $\boldsymbol{k}^{(m)}$ and $\boldsymbol{v}^{(m)}$ be as earlier. Assume that $\alpha_{j}=O\left(\frac{1}{g}\right)$ for all $j$. Then for $n$ large,

$\frac{1}{\left|\mathcal{H}_{n}\right|} \sum_{D \in \mathcal{H}_{n}}\left|\mathcal{L}\left(\frac{v_{1}}{q^{\frac{1}{2}+\alpha_{1}}}, \chi_{D}\right)\right|^{2 k_{1}} \ldots\left|\mathcal{L}\left(\frac{v_{m}}{q^{\frac{1}{2}+\alpha_{m}}}, \chi_{D}\right)\right|^{2 k_{m}} \gg_{\boldsymbol{k}^{(m)}, \boldsymbol{v}^{(m)}} \exp \left(\mu\left(\boldsymbol{v}^{(m)}, g\right)+\frac{1}{2} \sigma\left(\boldsymbol{v}^{(m)}, g\right)\right)$, where $\mu\left(\boldsymbol{v}^{(m)}, g\right)$ and $\sigma\left(\boldsymbol{v}^{(m)}, g\right)$ are defined by (1.5) and (1.6) respectively.

\footnotetext{
${ }^{1}$ The conjectural order of magnitude of these $L$-functions in the hyperelliptic ensemble can be compared with the autocorrelation of the random matrix polynomials (for example, see [[10, Eqs. (3.6) and (4.19)]).
} 
We also establish an upper bound of nearly the conjectured order of magnitude for the sum $\mathcal{S}_{n}\left(\boldsymbol{v}^{(m)}, \boldsymbol{k}^{(m)}\right)$

Theorem 1.5. With the assumption as in the Theorem 1.4, for any $\epsilon>0$,

$\frac{1}{\left|\mathcal{H}_{n}\right|} \sum_{D \in \mathcal{H}_{n}}\left|\mathcal{L}\left(\frac{v_{1}}{q^{\frac{1}{2}+\alpha_{1}}}, \chi_{D}\right)\right|^{2 k_{1}} \ldots\left|\mathcal{L}\left(\frac{v_{m}}{q^{\frac{1}{2}+\alpha_{m}}}, \chi_{D}\right)\right|^{2 k_{m}} \ll_{\boldsymbol{k}^{(m)}, \varepsilon} n^{\varepsilon} \exp \left(\mu\left(\boldsymbol{v}^{(m)}, g\right)+\frac{1}{2} \sigma\left(\boldsymbol{v}^{(m)}, g\right)\right)$,

where $\mu\left(\boldsymbol{v}^{(m)}, g\right)$ and $\sigma\left(\boldsymbol{v}^{(m)}, g\right)$ are defined by (1.5) and (1.6) respectively.

Theorem 1.5 is also true for any fixed sequence $\mathbf{k}^{(\mathbf{m})}$ of positive real numbers. Let us define $\mathcal{L}^{(l)}\left(u, \chi_{D}\right)$ as the $l$-th derivative of $\mathcal{L}\left(u, \chi_{D}\right)$. As an important consequence of Theorem 1.5, we have the following upper bound.

Theorem 1.6. Let $l \in \mathbb{N}$ and $\varepsilon>0$. For $n$ large, we have

$$
\sum_{D \in \mathcal{H}_{n}}\left|\mathcal{L}^{(l)}\left(q^{-1 / 2}, \chi_{D}\right)\right|^{k} \ll_{k, l, \varepsilon}\left|\mathcal{H}_{n}\right| g^{\frac{1}{2} k(k+1)+l k+\varepsilon} .
$$

1.3.2. Applications. From the Theorem 1.5 and in light of (2.3) if we specialize $n$ as $n=2 g+1$ and $\alpha_{j}$ 's are zero then we recover Theorem 1.2 .

The case of the mean value for $\mathcal{L}\left(q^{-1 / 2}, \chi_{D}\right)$ taken over $\mathcal{H}_{2 g+2}$ was investigated by Jung [18]. Taking $n=2 g+2$, we have the following corollary which generalizes the Theorem 1.2 :

Corollary 1.7. Let $\varepsilon>0$. For $n$ large, we have

$$
\frac{1}{\left|\mathcal{H}_{2 g+2}\right|} \sum_{D \in \mathcal{H}_{2 g+2}}\left|\mathcal{L}\left(q^{-1 / 2}, \chi_{D}\right)\right|^{k} \ll_{k, \varepsilon} g^{\frac{1}{2} k(k+1)+\varepsilon}
$$

Similarly, Theorem [1.6 provide upper bound for $k$-th moment of $\mathcal{L}^{(m)}\left(q^{-1 / 2}, \chi_{D}\right)$ with $D \in \mathcal{H}_{2 g+1}$ and $D \in \mathcal{H}_{2 g+2}$. More precisely,

Corollary 1.8. Let $l \in \mathbb{N}$ and $\varepsilon>0$. For $n$ large, we have

$$
\begin{gathered}
\sum_{D \in \mathcal{H}_{2 g+1}}\left|\mathcal{L}^{(l)}\left(q^{-1 / 2}, \chi_{D}\right)\right|^{k} \ll_{k, l, \varepsilon} q^{2 g+1} g^{\frac{1}{2} k(k+1)+l k+\varepsilon}, \\
\sum_{D \in \mathcal{H}_{2 g+2}}\left|\mathcal{L}^{(l)}\left(q^{-1 / 2}, \chi_{D}\right)\right|^{k} \ll_{k, l, \varepsilon} q^{2 g+2} g^{\frac{1}{2} k(k+1)+l k+\varepsilon} .
\end{gathered}
$$

Theorem 1.4 and 1.5 can be related to the main results of [7] and [25]. In fact we can say that $\mathcal{L}\left(\frac{v_{1}}{q^{\frac{1}{2}+\alpha_{1}}}, \chi_{D}\right)$ and $\mathcal{L}\left(\frac{v_{2}}{q^{\frac{1}{2}+\alpha_{2}}}, \chi_{D}\right)$ are essentially correlated when $\left|\theta_{j}\right| \asymp \frac{1}{g}$ for $j=1,2$ and independent when one of $\theta_{j}$ 's is much larger than $\frac{1}{g}$. More precisely, we have the following corollaries.

Corollary 1.9. Let $W$ and $W^{c}$ be defined by (1.7). For every $\varepsilon>0$ and $n$ large,

$$
\sum_{D \in \mathcal{H}_{n}}\left|\mathcal{L}\left(\frac{v_{1}}{q^{\frac{1}{2}+\alpha_{1}}}, \chi_{D}\right) \mathcal{L}\left(\frac{v_{2}}{q^{\frac{1}{2}+\alpha_{2}}}, \chi_{D}\right)\right|^{2 k}
$$




$$
\ll_{k, \varepsilon} \begin{cases}\left|\mathcal{H}_{n}\right| g^{2 k(4 k+1)+\varepsilon} & \text { if }|W|=2, \\ \frac{\left|\mathcal{H}_{n}\right| g^{3 k^{2}+k+\varepsilon}}{\left|\theta_{2}\right|^{k^{2}+k}\left|\theta_{1}-\theta_{2}\right|^{2 k^{2}}\left|\theta_{1}+\theta_{2}\right|^{2 k^{2}}} & \text { if } W=\{1\}, W^{c}=\{2\}, \\ \frac{\left|\mathcal{H}_{n}\right| g^{2 k^{2}+\varepsilon}}{\left|\theta_{1} \theta_{2}\right|^{k^{2}+k}\left|\theta_{1}-\theta_{2}\right|^{2 k^{2}}\left|\theta_{1}+\theta_{2}\right|^{2 k^{2}}} & \text { if }\left|W^{c}\right|=2, \lim _{g \rightarrow \infty} g\left|\theta_{1}-\theta_{2}\right|=\infty, \\ \frac{\left|\mathcal{H}_{n}\right| g^{4 k^{2}+\varepsilon}}{\left|\theta_{1} \theta_{2}\right|^{k^{2}+k}\left|\theta_{1}+\theta_{2}\right|^{2 k^{2}}} & \text { if }\left|W^{c}\right|=2, \lim _{g \rightarrow \infty} g\left|\theta_{1}-\theta_{2}\right|<\infty,\end{cases}
$$

Corollary 1.10. With the assumption as in Corollary [1.9, for $n$ large, we have

$$
\begin{aligned}
& \sum_{D \in \mathcal{H}_{n}}\left|\mathcal{L}\left(\frac{v_{1}}{q^{\frac{1}{2}+\alpha_{1}}}, \chi_{D}\right) \mathcal{L}\left(\frac{v_{2}}{q^{\frac{1}{2}+\alpha_{2}}}, \chi_{D}\right)\right|^{2 k} \\
& \gg_{k, \boldsymbol{u}^{(2)}} \begin{cases}\left|\mathcal{H}_{n}\right| g^{2 k(4 k+1)+\varepsilon} & \text { if }|W|=2, \\
\frac{\left|\mathcal{H}_{n}\right| g^{3 k^{2}+k+\varepsilon}}{\left|\theta_{2}\right|^{k^{2}+k}\left|\theta_{1}-\theta_{2}\right|^{2 k^{2}}\left|\theta_{1}+\theta_{2}\right|^{2 k^{2}}} & \text { if } W=\{1\}, W^{c}=\{2\},, \\
\frac{\left|\mathcal{H}_{n}\right| g^{2 k^{2}+\varepsilon}}{\left|\theta_{1} \theta_{2}\right|^{k^{2}+k}\left|\theta_{1}-\theta_{2}\right|^{2 k^{2}}\left|\theta_{1}+\theta_{2}\right|^{2 k^{2}}} & \text { if }\left|W^{c}\right|=2, \lim _{g \rightarrow \infty} g\left|\theta_{1}-\theta_{2}\right|=\infty, \\
\frac{\left|\mathcal{H}_{n}\right| g^{4 k^{2}+\varepsilon}}{\left|\theta_{1} \theta_{2}\right|^{2}+k\left|\theta_{1}+\theta_{2}\right|^{2 k^{2}}} & \text { if }\left|W^{c}\right|=2, \lim _{g \rightarrow \infty} g\left|\theta_{1}-\theta_{2}\right|<\infty .\end{cases}
\end{aligned}
$$

Remark 2. Corollary 1.9 and 1.10 gives the lower and upper bound over all monic square-free polynomials of both even and odd degree near the critical line.

\section{BACKGROUND FOR $L$-FUnCTIONS OVER FUNCTION FIELDS}

We begin this section with some preliminaries of $L$-functions over function fields. We will use [28] as a general reference.

2.1. Basic facts on $\mathbb{F}_{q}[t]$. We start by fixing a finite field $\mathbb{F}_{q}$ of odd cardinality $q=p^{r}, r \geq 1$ with a prime $p$. We denote by $\mathbb{A}=\mathbb{F}_{q}[t]$ the polynomial ring over $\mathbb{F}_{q}$. For a polynomial $f$ in $\mathbb{F}_{q}[t]$, it's degree will be denoted by either $\operatorname{deg}(f)$ or $d(f)$.

The set of all monic polynomials and monic irreducible polynomials of degree $n$ are denoted by $\mathcal{M}_{n, q}$ (or simply $\mathcal{M}_{n}$ as we fix $q$ ) and $\mathcal{P}_{n, q}$ (or simply $\mathcal{P}_{n}$ ) respectively. Let $\mathcal{M}=\cup_{n \geq 1} \mathcal{M}_{n}$ and $\mathcal{P}=\cup_{n \geq 1} \mathcal{P}_{n}$. we also denote the set of all monic polynomials and monic irreducible polynomials of degree less or equal to $n$ by $\mathcal{M}_{\leq n, q}$ (or simply $\mathcal{M}_{\leq n}$ ) and $\mathcal{P}_{\leq n, q}$ (or simply $\mathcal{P}_{\leq n}$ ) respectively. Let $\mathcal{H}_{n}$ denotes the set of monic square-free polynomials of degree $n$. Observe that for $n \geq 1,\left|\mathcal{M}_{n}\right|=q^{n}$ and

$$
\left|\mathcal{H}_{n}\right|= \begin{cases}q, & \text { if } n=1, \\ q^{n-1}(q-1), & \text { if } n \geq 2 .\end{cases}
$$

If $f$ is is a non-zero polynomial $\mathbb{F}_{q}[t]$, we define the norm of $f$ to be $|f|=q^{d(f)}$. If $f=0$, we set $|f|=0$. The prime polynomial theorem (see [28], Theorem 2.2) states that

$$
\left|\mathcal{P}_{n, q}\right|=\frac{q^{n}}{n}+O\left(\frac{q^{\frac{n}{2}}}{n}\right) .
$$

The zeta function of $\mathbb{A}$, denoted by $\zeta_{\mathbb{A}}(s)$ and is defined by

$$
\zeta_{\mathbb{A}}(s):=\sum_{f \in \mathcal{M}} \frac{1}{|f|^{s}}=\prod_{P \in \mathcal{P}}\left(1-|P|^{-s}\right)^{-1}, \quad \Re(s)>1 .
$$


One can easily prove that $\zeta_{\mathbb{A}}(s)=\frac{1}{1-q^{1-s}}$, and this provides an analytic continuation of zeta function to the complex plane with a simple pole at $s=1$. Using the change of variable $u=q^{-s}$,

$$
\mathcal{Z}(u)=\sum_{f \in \mathcal{M}} u^{d(f)}=\frac{1}{1-q u}, \quad \text { if }|u|<\frac{1}{q} .
$$

2.2. Quadratic Dirichlet character and properties of their $L$-functions. For a monic irreducible polynomial $P$, the quadratic residue symbol $\left(\frac{f}{P}\right)$ is defined by

$$
\left(\frac{f}{P}\right)= \begin{cases}1, & \text { if } f \text { is a square }(\bmod P), P \nmid f \\ -1, & \text { if } f \text { is not a square }(\bmod P), P \nmid f \\ 0, & \text { if } P \mid f .\end{cases}
$$

For monic square-free polynomial $D \in \mathbb{F}_{q}[t]$, the symbol $\left(\frac{D}{\text { }}\right)$ is defined by extending the above residue symbol multiplicatively. We denote the quadratic Dirichlet character $\chi_{D}$ by

$$
\chi_{D}(f)=\left(\frac{D}{f}\right) .
$$

The $L$-function associated to the quadratic Dirichlet character $\chi_{D}$ is defined by

$$
L\left(s, \chi_{D}\right)=\sum_{f \in \mathcal{M}} \frac{\chi_{D}(f)}{|f|^{s}}=\prod_{P \in \mathcal{P}}\left(1-\chi_{D}(P)|P|^{-s}\right)^{-1}, \Re(s)>1 .
$$

Using the change of variable $u=q^{-s}$, we have

$$
\mathcal{L}\left(u, \chi_{D}\right)=\sum_{f \in \mathcal{M}} \chi_{D}(f) u^{d(f)}=\prod_{P \in \mathcal{P}}\left(1-\chi_{D}(P) u^{d(P)}\right)^{-1}, \quad|u|<\frac{1}{q} .
$$

By [[28], Proposition 4.3], we see that if $n \geq d(D)$ then

$$
\sum_{f \in \mathcal{M}_{n}} \chi_{D}(f)=0
$$

It implies that $\mathcal{L}\left(u, \chi_{D}\right)$ is a polynomial of degree at most $d(D)-1$. From [29], $\mathcal{L}\left(u, \chi_{D}\right)$ has a trivial zero at $u=1$ if and only if $d(D)$ is even. This allows us to define the completed $L$-function as

$$
L\left(s, \chi_{D}\right)=\mathcal{L}\left(u, \chi_{D}\right)=(1-u)^{\lambda} \mathcal{L}^{*}\left(u, \chi_{D}\right)=\left(1-q^{-s}\right)^{\lambda} L^{*}\left(s, \chi_{D}\right),
$$

where

$$
\lambda= \begin{cases}1, & \text { if } d(D) \text { even } \\ 0, & \text { if } d(D) \text { odd }\end{cases}
$$

and $\mathcal{L}^{*}\left(u, \chi_{D}\right)$ is a polynomial of degree

$$
2 g=d(D)-1-\lambda
$$

satisfying the functional equation

$$
\mathcal{L}^{*}\left(u, \chi_{D}\right)=\left(q u^{2}\right)^{g} \mathcal{L}^{*}\left(\frac{1}{q u}, \chi_{D}\right)
$$

Because $\mathcal{L}$ and $\mathcal{L}^{*}$ are polynomial in $u$, it is convenient to define

$$
L^{*}\left(s, \chi_{D}\right)=\mathcal{L}^{*}\left(u, \chi_{D}\right)
$$


so that the above functional equation can be rewritten as

$$
L^{*}\left(s, \chi_{D}\right)=q^{(1-2 s) g} L^{*}\left(1-s, \chi_{D}\right) .
$$

The Riemann hypothesis for curve over finite fields, established by Weil [35], asserts that all the non-trivial zero of $\mathcal{L}^{*}\left(u, \chi_{D}\right)$ are lie on the circle $|u|=q^{-1 / 2}$, i.e,

$$
\mathcal{L}^{*}\left(u, \chi_{D}\right)=\prod_{j=1}^{2 g}\left(1-u \nu_{j}\right) \text { with }\left|\nu_{j}\right|=\sqrt{q} \text { for all } j .
$$

One can define the completed $L$-function in the following way. Set

$$
X_{D}(s)=|D|^{\frac{1}{2}-s} X(s),
$$

where

$$
X(s)= \begin{cases}q^{s-\frac{1}{2}}, & \text { if } d(D) \text { odd } \\ \frac{1-q^{-s}}{1-q^{-(1-s)}} q^{-1+2 s}, & \text { if } d(D) \text { even }\end{cases}
$$

Let us consider

$$
\Lambda\left(s, \chi_{D}\right)=L\left(s, \chi_{D}\right) X_{D}(s)^{-\frac{1}{2}} .
$$

Then $\Lambda\left(s, \chi_{D}\right)$ satisfies the symmetric functional equation

$$
\Lambda\left(s, \chi_{D}\right)=\Lambda\left(1-s, \chi_{D}\right) .
$$

2.3. Spectral Interpretation. Let $C$ be a non-singular projective curve over $\mathbb{F}_{q}$ of genus $g$. For each extension field of degree $k$ of $\mathbb{F}_{q}$, denote by $N_{k}(C)$ the number of points of $C$ in $\mathbb{F}_{q^{k}}$. Then, the zeta function associated to $C$ defined as

$$
Z_{C}(u)=\exp \left(\sum_{k=1}^{\infty} N_{k}(C) \frac{u^{k}}{k}\right), \quad|u|<\frac{1}{q},
$$

is known to be a rational function of $u$ of the form

$$
Z_{C}(u)=\frac{P_{C}(u)}{(1-u)(1-q u)} .
$$

Additionally, we know that $P_{C}(u)$ is a polynomial of degree $2 g$ with integer coefficients, satisfying a functional equation

$$
P_{C}(u)=\left(q u^{2}\right)^{g} P_{C}\left(\frac{1}{q u}\right) .
$$

The Riemann Hypothesis, proved by Weil [35], says that the zeros of $P_{C}(u)$ all lie on the circle $|u|=\frac{1}{\sqrt{q}}$. Thus one may give a spectral interpretation of $P_{C}(u)$ as the characteristic polynomial of a $2 g \times 2 g$ unitary matrix $\Theta_{C}$ :

$$
P_{C}(u)=\operatorname{det}\left(I-u \sqrt{q} \Theta_{C}\right) .
$$

Thus the eigenvalues $e^{i \theta_{j}}$ of $\Theta_{C}$ correspond to the zeros, $q^{-1 / 2} e^{-i \theta_{j}}$, of $Z_{C}(u)$. The matrix $\Theta_{C}$ is called the unitarized Frobenius class of $C$.

To put this in the context of our case, note that, for a family of hyperelliptic curves $C_{D}$ : $y^{2}=D(t)$ of genus $g$, the numerator of the zeta function $Z_{C}(u)$ associated to $C_{D}$ is coincide with the $L$-function $\mathcal{L}^{*}\left(u, \chi_{D}\right)$, i.e., $P_{C}(u)=\mathcal{L}^{*}\left(u, \chi_{D}\right)$. 


\section{Preliminary Lemmas}

We start with an analog of approximate functional equation for $L\left(s, \chi_{D}\right)$. Recall that $2 g=n-1-\lambda$ where $\lambda$ is defined as in (2.2).

Lemma 3.1 (Approximate functional equation). Let $\chi_{D}$ be a quadratic Dirichlet character, where $D \in \mathcal{H}_{n}$. Then for $1 / 2 \leq s<1$,

$$
\begin{aligned}
L\left(s, \chi_{D}\right) & =\sum_{f \in \mathcal{M}_{\leq g}} \frac{\chi_{D}(f)}{|f|^{s}}+X_{D}(s) \sum_{f \in \mathcal{M}_{\leq g-1}} \frac{\chi_{D}(f)}{|f|^{1-s}} \\
& -\lambda q^{-s(g+1)} \sum_{f \in \mathcal{M}_{\leq g}} \chi_{D}(f)-\lambda X_{D}(s) q^{-(1-s) g} \sum_{f \in \mathcal{M}_{\leq g-1}} \chi_{D}(f),
\end{aligned}
$$

where $X_{D}(s)$ is defined by (2.4) respectively.

Proof. The case $s=\frac{1}{2}$ is proved in [2] for $D \in \mathcal{H}_{2 g+1}$ and [18] for $D \in \mathcal{H}_{2 g+2}$. Their methods can be easily generalized for any $s \in(1 / 2,1)$.

The following lemma gives an asymptotic formula for a square polynomial in hyperelliptic ensemble.

Lemma 3.2. For $f \in \mathcal{M}$, we have

$$
\frac{1}{\left|\mathcal{H}_{n}\right|} \sum_{D \in \mathcal{H}_{n}} \chi_{D}\left(f^{2}\right)=\prod_{\substack{P \in \mathcal{P} \\ P \mid f}}\left(1+\frac{1}{|P|}\right)^{-1}+O\left(\left|\mathcal{H}_{n}\right|^{-1}\right) .
$$

Proof. See [[5], Lemma 3.7] for $n=2 g+1$. To get the result for $n=2 g+2$, it is a small adaptation of their proof.

The following lemma is an analog of Polya-Vinogradov inequality over function fields.

Lemma 3.3 (Polya-Vinogradov inequality). For $l \in \mathcal{M}$ not a perfect square, let $l=l_{1} l_{2}{ }^{2}$ with $l_{1}$ square-free. Then for any $\epsilon>0$,

$$
\left|\sum_{D \in \mathcal{H}_{n}} \chi_{D}(l)\right| \ll_{\varepsilon} \sqrt{\left|\mathcal{H}_{n}\right|}\left|l_{1}\right|^{\epsilon} .
$$

Proof. One can easily generalize the above inequality which was proved in [6], Lemma 3.5] for $n=2 g+1$. Here we give a different proof in the above form for completeness.

First assume that $l_{1}=P_{1} P_{2} \ldots P_{k}$, where $P_{j}$ 's are distinct prime polynomials, and $\operatorname{deg}\left(l_{1}\right) \leq$ $n$. Similar to the proof of Lemma 3.5 in [5], which in particular case $k=2$, one can show:

$$
\left|\sum_{D \in \mathcal{H}_{n}} \chi_{D}(l)\right|=\left|\sum_{D \in \mathcal{H}_{n}} \chi_{D}\left(l_{1}\right)\right| \leq \frac{q g^{k-1}\left(d\left(P_{1}\right)+\ldots+d\left(P_{k}\right)\right)}{d\left(P_{1}\right) \ldots d\left(P_{k}\right)}\left|l_{1}\right|^{\frac{1}{2}} \ll_{\epsilon} \sqrt{\left|\mathcal{H}_{n}\right|}\left|l_{1}\right|^{\epsilon} .
$$

Finally let $\operatorname{deg}\left(l_{1}\right)>n$. We combine Lemma 3.1 of [6] and Lemma 3.5 of [5] to obtain

$$
\left|\sum_{D \in \mathcal{H}_{n}} \chi_{D}\left(l_{1}\right)\right| \ll_{\epsilon} \sqrt{\left|\mathcal{H}_{n}\right|}\left|l_{1}\right|^{\epsilon} .
$$

The following lemma gives an upper bound for the logarithm of $\mathcal{L}\left(u, \chi_{D}\right)$ inside the critical region. 
Lemma 3.4. Let $0 \leq \alpha \leq \frac{1}{2}, v=e^{i \theta}, \theta \in[0, \pi)$ and $N$ be a positive integer. Then for $D \in \mathcal{H}_{n}$,

$$
\log \left|\mathcal{L}\left(\frac{v}{q^{\frac{1}{2}+\alpha}}, \chi_{D}\right)\right| \leq \frac{2 g}{N+1} \log \left(\frac{1+q^{-\alpha(N+1)}}{1+q^{-2(N+1)}}\right)+\Re \sum_{d(f) \leq N} \frac{a_{\alpha}(d(f)) \chi_{D}(f) \Lambda(f) v^{d(f)}}{|f|^{\frac{1}{2}}}+O(1),
$$

where

$$
a_{\alpha}(d(f))=\frac{1}{d(f)|f|^{\alpha}}-\frac{1}{d(f)|f|^{2}}+O\left(\frac{1}{(N+1) q^{(N+1) \alpha}}\right), \quad \text { for } 1 \leq d(f) \leq N .
$$

Proof. From the functional equation (2.6), we observe that

$$
\left|\Lambda\left(\alpha+i t, \chi_{D}\right)\right|=\left|\frac{\Lambda\left(\frac{5}{2}-i t, \chi_{D}\right) \Lambda\left(1-\alpha-i t, \chi_{D}\right)}{\Lambda\left(-\frac{3}{2}+i t, \chi_{D}\right)}\right|=\frac{\left|\Lambda\left(\frac{5}{2}-i t, \chi_{D}\right)\right|\left|\Lambda\left(1-\alpha+i t, \chi_{D}\right)\right|}{\left|\Lambda\left(-\frac{3}{2}+i t, \chi_{D}\right)\right|} .
$$

Recall that

$$
L\left(\alpha+i t, \chi_{D}\right)=\left(1-q^{-\alpha-i t}\right)^{\lambda} L^{*}\left(\alpha+i t, \chi_{D}\right) .
$$

Note that $\left|L^{*}\left(\frac{5}{2}-i t, \chi_{D}\right)\right| \sim 1$. Using the expression (2.5) for $\Lambda\left(s, \chi_{D}\right)$, we get

$$
\left|L\left(\alpha+i t, \chi_{D}\right)\right|=q^{g(5-2 \alpha)}\left|1-q^{-\alpha-i t}\right|^{\lambda} \prod_{j=1}^{2 g}\left(\frac{q^{2 \alpha-1}+1-2 q^{\alpha-\frac{1}{2}} \cos \left(2 \pi \theta_{j}-t \log q\right)}{q^{4}+1-2 q^{2} \cos \left(2 \pi \theta_{j}-t \log q\right)}\right)^{\frac{1}{2}}
$$

Since

$$
q^{2 \alpha-1}+1-2 q^{\alpha-\frac{1}{2}} \cos \left(2 \pi \theta_{j}-t \log q\right)=\left(q^{\alpha-\frac{1}{2}}-1\right)^{2}+4 q^{\alpha-\frac{1}{2}} \sin ^{2}\left(\pi \theta_{j}-\frac{t \log q}{2}\right)
$$

with a similar expression holding for the denominator, it follows that

$$
\log \left|L\left(\alpha+i t, \chi_{D}\right)\right|=g\left(\frac{5}{2}-\alpha\right) \log q-\frac{1}{2} \sum_{j=1}^{2 g} \log \left(\frac{a^{2}+\sin ^{2}\left(\pi \theta_{j}-\frac{t \log q}{2}\right)}{b^{2}+\sin ^{2}\left(\pi \theta_{j}-\frac{t \log q}{2}\right)}\right)+O(1),
$$

where

$$
a=\frac{q^{2}-1}{2 q}, b=\frac{q^{\alpha-\frac{1}{2}}-1}{2 q^{\frac{\alpha}{2}-\frac{1}{4}}} .
$$

The remaining part of the proof is the same as the proof of Lemma 8.1 in [14 proved by A. Florea.

Lemma 3.5. Let $\theta \in(-\pi, \pi)$, then we have $\sum_{m=1}^{n} \frac{\cos (\theta m)}{m} \leq \log \left(\min \left\{n, \frac{1}{|\theta|}\right\}\right)+O(1)$.

Proof. See [[14], Lemma 9.1].

Lemma 3.6. Let $k, y$ be integers such that $2 k y \leq n$. For any complex numbers $\{a(P)\}_{p \in \mathcal{P}}$, we have

$$
\sum_{D \in \mathcal{H}_{n}}\left|\sum_{d(P) \leq y} \frac{a(P) \chi_{D}(P)}{|P|^{\frac{1}{2}}}\right|^{2 k} \ll\left|\mathcal{H}_{n}\right| \frac{(2 k) !}{k ! 2^{k}}\left(\sum_{d(P) \leq y} \frac{|a(P)|^{2}}{|P|}\right)^{k} .
$$

Proof. This is an easy generalization of the Lemma 8.4 of [14] and Lemma 6.3 of [34]. 
During the study of our main theorems it seems interesting to estimate the following bounds for the zeta function over function fields. This is an analog of bounding the Riemann zeta function near to 1-line.

Lemma 3.7. Let $v=e^{i \theta}$, where $\theta \in(-\pi, \pi)$. Let $C$ be a circle of radius $\frac{\widetilde{r}}{g}$ centred at $\frac{1}{q}$, where

$$
\widetilde{r}=\lim _{g \rightarrow \infty} g|\theta|<\infty
$$

For any $u$ in $C$, we have

$$
\mathcal{Z}(u v) \ll g \quad \text { if } \lim _{g \rightarrow \infty} g|\theta|<\infty .
$$

For any $u$ such that $\left|u-\frac{1}{q}\right|=O(1 / g)$, we have

$$
\mathcal{Z}(u v) \ll \frac{1}{|\theta|} \text { if } \lim _{g \rightarrow \infty} g|\theta|=\infty .
$$

Proof. First assume that $\theta \in(-\pi, \pi)$ be such that $\lim _{g \rightarrow \infty} g|\theta|=\infty$. Then using $\left|u-\frac{1}{q}\right|=$ $O(1 / g)$, we have the following estimates:

$$
\left|\frac{v}{(1-v)}(1-q u)\right|=o(1)
$$

and

$$
\left|(1-v)^{-1}\right| \ll \frac{1}{|\theta|}
$$

Thus

$$
|\mathcal{Z}(u v)|=\left|(1-q u v)^{-1}\right|=\left|(1-v)^{-1}\left(1+\frac{v}{(1-v)}(1-q u)\right)^{-1}\right| \ll \frac{1}{|\theta|} .
$$

Finally let $\theta$ be such that $\lim _{g \rightarrow \infty} g|\theta|<\infty$. Then $\left|u-\frac{1}{q}\right| \leq \frac{\widetilde{r}}{g}$. We use the change of variable $u=q^{-s}$ to get the hypothesis of the form $|s-1| \leq \frac{\widetilde{r}}{g}$. Since

$$
\mathcal{Z}(u v)=\sum_{f \in \mathcal{M}}(u v)^{\operatorname{deg}(f)}
$$

it is enough to show that

$$
\sum_{f \in \mathcal{M}} \frac{1}{|f|^{1+\widetilde{r} / g-i \theta / \log q}}=O(g) .
$$

Therefore using Lemma 3.5 and the prime polynomial theorem, we obtain

$$
\begin{aligned}
& \log \left|\sum_{f \in \mathcal{M}} \frac{1}{|f|^{1+\widetilde{r} / g-i \theta / \log q} \mid}\right|=\Re \sum_{P \in \mathcal{P}} \frac{1}{|P|^{1+\widetilde{r} / g-i \theta / \log q}}+O(1)=\Re \sum_{n} \frac{1}{n q^{n(\widetilde{r} / g-i \theta / \log q)}}+O(1) \\
&=\Re \sum_{n \leq g} \frac{q^{\frac{i n \theta}{\log q}}}{n}-\Re \sum_{n \leq g}\left(\frac{1}{n}-\frac{1}{n q^{\frac{\widetilde{r}}{g}}}\right) q^{\frac{i n \theta}{\log q}}+\Re \sum_{n>g} \frac{q^{\frac{i n \theta}{\log q}}}{n q^{\frac{\widetilde{r}}{g}}}+O(1) \\
&=\sum_{n \leq g} \frac{\cos (n \theta)}{n}+O(1) \leq \log \left(\min \left\{g, \frac{1}{|\theta|}\right\}\right) \leq \log g,
\end{aligned}
$$

and the lemma's proof is concluded. 


\section{Proof of Theorem 1.3}

Throughout this section, for the sake of simplicity, we write $\boldsymbol{v}^{(2)}$ and $\boldsymbol{k}^{(2)}$ simply as $\boldsymbol{v}$ and $\boldsymbol{k}$ respectively. For any $k_{1}, k_{2} \in \mathbb{N}$, we write

$$
\mathcal{L}\left(\frac{v_{1}}{q^{1 / 2+\alpha_{1}}}, \chi_{D}\right)^{k_{1}} \mathcal{L}\left(\frac{v_{2}}{q^{1 / 2+\alpha_{2}}}, \chi_{D}\right)^{k_{2}}=\sum_{f \in \mathcal{M}} a_{f} \frac{\chi_{D}(f)}{|f|^{1 / 2}}
$$

where

$$
a_{f}=\sum_{f_{1} f_{2}=f} \frac{\tau_{k_{1}\left(f_{1}\right)} \tau_{k_{2}\left(f_{2}\right)}}{\left|f_{1}\right|^{\alpha_{1}}\left|f_{2}\right|^{\alpha_{2}}} e^{i\left(\theta_{1} d\left(f_{1}\right)+\theta_{2} d\left(f_{2}\right)\right)} .
$$

We start by defining the following truncated $L$-function which is an analog of Dirichlet polynomials over number fields:

$$
\mathcal{L}_{\leq\left(k_{1}+k_{2}\right) X}\left(\boldsymbol{v}, \chi_{D}\right):=\sum_{f \in \mathcal{M}_{\leq\left(k_{1}+k_{2}\right) X}} a_{f} \frac{\chi_{D}(f)}{|f|^{1 / 2}},
$$

where $a_{f}$ is defined by (4.2) and the parameter $X$ will be chosen later. We call $X$ as point of truncation of (4.1).

Using Cauchy-Schwarz inequality, we have

$$
\begin{aligned}
& \sum_{D \in \mathcal{H}_{n}}\left|\mathcal{L}\left(\frac{v_{1}}{q^{1 / 2+\alpha_{1}}}, \chi_{D}\right)^{k_{1}} \mathcal{L}\left(\frac{v_{2}}{q^{1 / 2+\alpha_{2}}}, \chi_{D}\right)^{k_{2}} \overline{\mathcal{L}_{\leq\left(k_{1}+k_{2}\right) X}\left(\boldsymbol{v}, \chi_{D}\right)}\right| \\
& \quad \leq\left(\sum_{D \in \mathcal{H}_{n}}\left|\mathcal{L}\left(\frac{v_{1}}{q^{1 / 2+\alpha_{1}}}, \chi_{D}\right)^{k_{1}} \mathcal{L}\left(\frac{v_{2}}{q^{1 / 2+\alpha_{2}}}, \chi_{D}\right)^{k_{2}}\right|^{2}\right)^{1 / 2}\left(\sum_{D \in \mathcal{H}_{n}}\left|\overline{\mathcal{L}_{\leq\left(k_{1}+k_{2}\right) X}\left(\boldsymbol{v}, \chi_{D}\right)}\right|^{2}\right)^{1 / 2} .
\end{aligned}
$$

Therefore, we obtain

$$
\sum_{D \in \mathcal{H}_{n}}\left|\mathcal{L}\left(\frac{v_{1}}{q^{1 / 2+\alpha_{1}}}, \chi_{D}\right)^{k_{1}} \mathcal{L}\left(\frac{v_{2}}{q^{1 / 2+\alpha_{2}}}, \chi_{D}\right)^{k_{2}}\right|^{2} \geq \frac{S_{1}^{2}}{S_{2}}
$$

where

$$
S_{1}:=\sum_{D \in \mathcal{H}_{n}}\left|\mathcal{L}\left(\frac{v_{1}}{q^{1 / 2+\alpha_{1}}}, \chi_{D}\right)^{k_{1}} \mathcal{L}\left(\frac{v_{2}}{q^{1 / 2+\alpha_{2}}}, \chi_{D}\right)^{k_{2}} \overline{\mathcal{L}_{\leq\left(k_{1}+k_{2}\right) X}\left(\boldsymbol{v}, \chi_{D}\right)}\right|
$$

and

$$
S_{2}:=\sum_{D \in \mathcal{H}_{n}}\left|\overline{\mathcal{L}_{\leq\left(k_{1}+k_{2}\right) X}\left(\boldsymbol{v}, \chi_{D}\right)}\right|^{2} .
$$

Now we establish an asymptotic formula for $S_{2}$ and a lower bound for $S_{1}$.

4.1. Estimation of the sum $S_{2}$. Inserting the $D$-sum after expanding square in $S_{2}$, we get

$$
S_{2}=\sum_{f \in \mathcal{M}_{\leq\left(k_{1}+k_{2}\right) X}} \sum_{f^{\prime} \in \mathcal{M}_{\leq\left(k_{1}+k_{2}\right) X}} \frac{a_{f} \overline{a_{f^{\prime}}}}{\left|f f^{\prime}\right|^{1 / 2}} \sum_{D \in \mathcal{H}_{n}} \chi_{D}\left(f f^{\prime}\right) .
$$


Case 1. Assume that $f f^{\prime} \neq \square$. Observe that $a_{f} \ll_{\varepsilon}|f|^{\varepsilon}$ and using Lemma 3.3, we obtain that

$$
S_{2} \ll \sqrt{\left|\mathcal{H}_{n}\right|} \sum_{f \in \mathcal{M}_{\leq 2\left(k_{1}+k_{2}\right) X}} \frac{1}{|f|^{\frac{1}{2}-\varepsilon}} \ll \sqrt{\left|\mathcal{H}_{n}\right|} q^{2\left(\frac{1}{2}+\varepsilon\right)\left(k_{1}+k_{2}\right) X} .
$$

Let us choose $X=\frac{g}{2\left(k_{1}+k_{2}\right)}$ 2. So, we have

$$
S_{2} \ll q^{\left(\frac{3}{2}+\varepsilon\right) g} .
$$

Case 2. Assume that $f f^{\prime}=\square=l^{2}$, where $l \in \mathbb{F}_{q}[t]$. By using Lemma 3.2 and $\tau_{k}(f) \ll_{\varepsilon}|f|^{\varepsilon}$,

$$
\begin{aligned}
& S_{2}=\left|\mathcal{H}_{n}\right| \sum_{l \in \mathcal{M}_{\leq\left(k_{1}+k_{2}\right) X}} \frac{1}{|l|} \sum_{f_{1} f_{2} f_{3} f_{4}=l^{2}} \frac{\tau_{k_{1}\left(f_{1}\right)} \tau_{k_{1}\left(f_{2}\right)} \tau_{k_{2}\left(f_{3}\right)} \tau_{k_{2}\left(f_{4}\right)}}{\left|f_{1} f_{2}\right|^{\alpha_{1}}\left|f_{3} f_{4}\right|^{\alpha_{2}}} \\
& \times e^{i \theta_{1}\left(d\left(f_{1}\right)-d\left(f_{2}\right)\right)+i \theta_{2}\left(d\left(f_{3}\right)-d\left(f_{4}\right)\right)} \prod_{P \mid l}\left(1+\frac{1}{|P|}\right)^{-1} \\
& +O\left(\sum_{l \in \mathcal{M}_{\leq\left(k_{1}+k_{2}\right) X}} \frac{1}{|l|} \sum_{f_{1} f_{2} f_{3} f_{4}=l^{2}} \frac{\tau_{k_{1}\left(f_{1}\right)} \tau_{k_{1}\left(f_{2}\right)} \tau_{k_{2}\left(f_{3}\right)} \tau_{k_{2}\left(f_{4}\right)}}{\left|f_{1} f_{2}\right|^{\alpha_{1}}\left|f_{3} f_{4}\right|^{\alpha_{2}}}\right) \\
& =\left|\mathcal{H}_{n}\right| \sum_{l \in \mathcal{M}_{\leq\left(k_{1}+k_{2}\right) X}} \frac{b(l)}{|l|}+O\left(q^{\varepsilon\left(k_{1}+k_{2}\right) X}\right) \text {, }
\end{aligned}
$$

where

$$
b(l)=\sum_{f_{1} f_{2} f_{3} f_{4}=l^{2}} \frac{\tau_{k_{1}\left(f_{1}\right)} \tau_{k_{1}\left(f_{2}\right)} \tau_{k_{2}\left(f_{3}\right)} \tau_{k_{2}\left(f_{4}\right)}}{\left|f_{1} f_{2}\right|^{\alpha_{1}}\left|f_{3} f_{4}\right|^{\alpha_{2}}} e^{i \theta_{1}\left(d\left(f_{1}\right)-d\left(f_{2}\right)\right)+i \theta_{2}\left(d\left(f_{3}\right)-d\left(f_{4}\right)\right)} \prod_{P \mid l}\left(1+\frac{1}{|P|}\right)^{-1} .
$$

We use the Perron's formula $3^{3}$ to get

$$
\sum_{l \in \mathcal{M}_{\leq\left(k_{1}+k_{2}\right) X}} \frac{b(l)}{|l|}=\frac{1}{2 \pi i} \int_{|u|=r} B(u) \frac{(q u)^{-\left(k_{1}+k_{2}\right) X}}{(1-q u)} \frac{d u}{u},
$$

where

$$
B(u)=\sum_{l \in \mathcal{M}} b(l) u^{d(l)} \quad \text { and } \quad r<\frac{1}{q} .
$$

For an irreducible polynomial $P$, we observe that

$b(P)=\left(1+\frac{1}{|P|}\right)^{-1} \sum_{f_{1} f_{2} f_{3} f_{4}=P^{2}} \frac{\tau_{k_{1}\left(f_{1}\right)} \tau_{k_{1}\left(f_{2}\right)} \tau_{k_{2}\left(f_{3}\right)} \tau_{k_{2}\left(f_{4}\right)}}{\left|f_{1} f_{2}\right|^{\alpha_{1}}\left|f_{3} f_{4}\right|^{\alpha_{2}}} e^{i \theta_{1}\left(d\left(f_{1}\right)-d\left(f_{2}\right)\right)+i \theta_{2}\left(d\left(f_{3}\right)-d\left(f_{4}\right)\right)}$

\footnotetext{
${ }^{2}$ For the Theorem 1.4 the point of truncation will be $\left(k_{1}+\ldots+k_{m}\right) X$ and the choice of $X$ is equal to $\frac{g}{2\left(k_{1}+\ldots+k_{m}\right)}$.

${ }^{3}$ Perron's formula in function fields comes through the Cauchy's integral formula. More precisely $\sum_{f \in \mathcal{M}_{\leq x}} a_{f}=\frac{1}{2 \pi i} \int_{|u|=r}\left(\sum_{f \in \mathcal{M}} a_{f} u^{\operatorname{deg}(f)}\right) \frac{d u}{u^{X+1}(1-u)}$, provided that the power series $\sum_{f \in \mathcal{M}} a_{f} u^{\operatorname{deg}(f)}$ is absolutely convergent in $|u| \leq r<1$.
} 


$$
=\left(1+\frac{1}{|P|}\right)^{-1}\left(\sum_{\substack{j=1 \\ \epsilon_{j} \in\{ \pm 1\}}}^{2} \frac{k_{j}\left(k_{j}+1\right)}{2} \frac{e^{2 i \epsilon_{j} \theta_{j} d(P)}}{|P|^{2 \alpha_{j}}}+\sum_{j=1}^{2} \frac{k_{j}^{2}}{|P|^{2 \alpha_{j}}}+\sum_{\epsilon_{j} \in\{ \pm 1\}} \frac{k_{1} k_{2}}{|P|^{\alpha_{1}+\alpha_{2}}} e^{i\left(\epsilon_{1} \theta_{1}+\epsilon_{2} \theta_{2}\right) d(P)}\right),
$$

which allows us to write $B(u)$ as

$$
B(u)=\prod_{j=1}^{2} \mathcal{Z}^{k_{j}^{2}}(u) \prod_{\substack{j=1 \\ \epsilon_{j} \in\{ \pm 1\}}}^{2} \mathcal{Z}^{\frac{k_{j}\left(k_{j}+1\right)}{2}}\left(u e^{2 i \epsilon_{j} \theta_{j}}\right) \prod_{\epsilon_{j} \in\{ \pm 1\}} \mathcal{Z}^{k_{1} k_{2}}\left(u e^{i\left(\epsilon_{1} \theta_{1}+\epsilon_{2} \theta_{2}\right)}\right) C(u) .
$$

Here $C(u)$ is absolutely convergent for $|u|<\frac{1}{\sqrt{q}}$. Therefore,

$$
\begin{aligned}
& \sum_{l \in \mathcal{M}_{\leq\left(k_{1}+k_{2}\right) X}} \frac{b(l)}{|l|}=\frac{1}{2 \pi i} \int_{|u|=r} B(u) \frac{(q u)^{-\left(k_{1}+k_{2}\right) X}}{(1-q u)} \frac{d u}{u} \\
&=\frac{1}{2 \pi i} \int_{|u|=r} \prod_{j=1}^{2} \mathcal{Z}^{k_{j}^{2}}(u) \prod_{\substack{j=1 \\
\epsilon_{j} \in\{ \pm 1\}}}^{2} \mathcal{Z}^{\frac{k_{j}\left(k_{j}+1\right)}{2}}\left(u e^{2 i \epsilon_{j} \theta_{j}}\right) \prod_{\epsilon_{j} \in\{ \pm 1\}} \mathcal{Z}^{k_{1} k_{2}}\left(u e^{i\left(\epsilon_{1} \theta_{1}+\epsilon_{2} \theta_{2}\right)}\right) \\
& \\
& \times C(u) \frac{(q u)^{-\left(k_{1}+k_{2}\right) X}}{(1-q u)} \frac{d u}{u}
\end{aligned}
$$

where $r=\frac{1}{q^{1+\varepsilon}}$.

4.1.1. Calculating the main term of $S_{2}$. To get main term we have to shift the contour of integration (4.4) over $u$ to a circle of radius $|u|=R=\frac{1}{q^{1 / 2}+\varepsilon}$. The integrand has a pole at $u=\frac{1}{q}$ of order $k_{1}^{2}+k_{2}^{2}+1$ and at $u=\frac{1}{q e^{2 i \epsilon_{j} \theta_{j}}}$ of order $\frac{k_{j}\left(k_{j}+1\right)}{2}$ and at $u=\frac{1}{q e^{i\left(\epsilon_{1} \theta_{1}+\epsilon_{2} \theta_{2}\right)}}$ of order $k_{1} k_{2}$, where $\epsilon_{j} \in\{ \pm 1\}$ and $j=1,2$.

We define

$$
D(u)=\prod_{j=1}^{2} \mathcal{Z}^{k_{j}^{2}}(u) \prod_{\substack{j=1 \\ \epsilon_{j} \in\{ \pm 1\}}}^{2} \mathcal{Z}^{\frac{k_{j}\left(k_{j}+1\right)}{2}}\left(u e^{2 i \epsilon_{j} \theta_{j}}\right) \prod_{\epsilon_{j} \in\{ \pm 1\}} \mathcal{Z}^{k_{1} k_{2}}\left(u e^{i\left(\epsilon_{1} \theta_{1}+\epsilon_{2} \theta_{2}\right)}\right) C(u) \frac{(q u)^{-\left(k_{1}+k_{2}\right) X}}{u(1-q u)}
$$

Using the Cauchy's residue theorem, we obtain

$$
\begin{aligned}
\frac{1}{2 \pi i} \int_{|u|=r} D(u) d u & =\frac{1}{2 \pi i} \int_{|u|=R} D(u) d u-\operatorname{Res}_{u=1 / q} D(u)-\sum_{\substack{j=1 \\
\epsilon_{j} \in\{ \pm 1\}}}^{2} \underset{u=1 / q e^{2 i \epsilon_{j} \theta_{j}}}{\operatorname{Res}} D(u) \\
& -\sum_{\epsilon_{j} \in\{ \pm 1\}} \operatorname{Res}_{u=1 / q e^{i\left(\epsilon_{1} \theta_{1}+\epsilon_{2} \theta_{2}\right)}} D(u),
\end{aligned}
$$

where $r=\frac{1}{q^{1+\varepsilon}}$ and $R=\frac{1}{q^{1 / 2+\varepsilon}}$.

\footnotetext{
${ }^{4}$ Cauchy's residue theorem says that if $\gamma$ is a simple closed, positively oriented contour in the complex

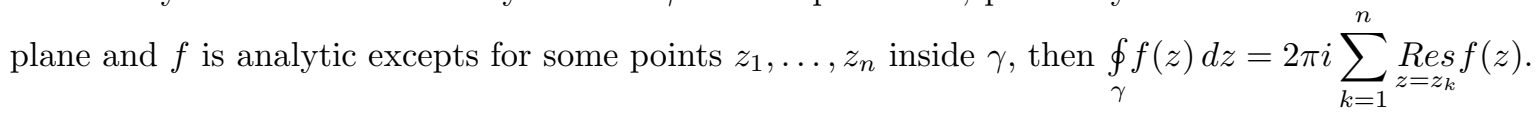


On the circle $|u|=R=\frac{1}{q^{1 / 2}+\varepsilon}$, we see that the functions $\frac{1}{1-q u}, \frac{1}{1-q u e^{i\left(\epsilon_{1} \theta_{1}+\epsilon_{2} \theta_{2}\right)}}$ and $\frac{1}{1-q u e^{2 i \epsilon_{j} \theta_{j}}}$ are bounded. This leads

$$
\frac{1}{2 \pi i} \int_{|u|=R} D(u) d u \ll q^{-\left(\frac{1}{2}-\varepsilon\right)\left(k_{1}+k_{2}\right) X}
$$

Evaluation of the sum of residues. We claim that

$$
\begin{aligned}
& \underset{u=1 / q}{\operatorname{Res}} D(u)+\sum_{\substack{j=1 \\
\epsilon_{j} \in\{ \pm 1\}}}^{2} \operatorname{Res}_{u=1 / q e^{2 i \epsilon_{j} \theta_{j}}} D(u)+\sum_{\epsilon_{j} \in\{ \pm 1\}} \operatorname{Res}_{u=1 / q e^{i\left(\epsilon_{1} \theta_{1}+\epsilon_{2} \theta_{2}\right)}} D(u) \\
& \sim_{\boldsymbol{k}, \widetilde{c}} g^{k_{1}^{2}+k_{2}^{2}} \prod_{j=1}^{2}\left(\min \left\{\frac{1}{2\left|\theta_{j}\right|}, g\right\}\right)^{k_{j}\left(k_{j}+1\right)}\left(\min \left\{\frac{1}{\left|\theta_{1}-\theta_{2}\right|}, g\right\}\right)^{2 k_{1} k_{2}}\left(\min \left\{\frac{1}{\left|\theta_{1}+\theta_{2}\right|}, g\right\}\right)^{2 k_{1} k_{2}}
\end{aligned}
$$

where

$$
\widetilde{c}:=\widetilde{c}_{\boldsymbol{v}}+1 \text { with } \widetilde{c}_{\boldsymbol{v}} \text { is defined as in (1.8). }
$$

Let us define the following sets

$$
\begin{aligned}
& W_{1}=\left\{j \in\{1,2\}: \lim _{g \rightarrow \infty} g\left|\theta_{j}\right|<\infty\right\}, \quad W_{1}^{c}=\left\{j \in\{1,2\}: \lim _{g \rightarrow \infty} g\left|\theta_{j}\right|=\infty\right\}, \\
& W_{2}=\left\{(1,2): \lim _{g \rightarrow \infty} g\left|\theta_{1}-\theta_{2}\right|<\infty\right\}, \quad W_{2}^{c}=\left\{(1,2): \lim _{g \rightarrow \infty} g\left|\theta_{1}-\theta_{2}\right|=\infty\right\}, \\
& W_{-2}=\left\{(1,2): \lim _{g \rightarrow \infty} g\left|\theta_{1}+\theta_{2}\right|<\infty\right\}, \quad W_{-2}^{c}=\left\{(1,2): \lim _{g \rightarrow \infty} g\left|\theta_{1}+\theta_{2}\right|=\infty\right\} .
\end{aligned}
$$

We call the elements of the sets $W_{1}$ and $W_{1}^{c}$ as finite and infinite "single shift" respectively. We also call the elements of the sets $W_{\epsilon 2}$ and $W_{\epsilon 2}^{c}, \epsilon \in\{1,-1\}$ as finite and infinite "pair shift" respectively5.

Estimation of finite "single shift" and "pair shift". Cauchy's residue theorem allows us to write

$$
\operatorname{Res}_{u=\frac{1}{q}} D(u)+\sum_{\substack{j \in W_{1} \\ \epsilon_{j} \in\{ \pm 1\}}} \operatorname{Res}_{u=\frac{1}{q e^{2 i \epsilon_{j} \theta_{j}}}} D(u)+\sum_{\substack{(1,2) \in W_{\epsilon 2} \\ \epsilon, \epsilon_{j} \in\{ \pm 1\}}} \operatorname{Res}_{\substack{1 \\ q e^{i\left(\epsilon_{1} \theta_{1}+\epsilon_{2} \theta_{2}\right)}}} D(u)=\int_{\Gamma} D(u) d u
$$

where $\Gamma$ is a circle centered at $\frac{1}{q}$ of radius $\frac{\widetilde{c}}{g}$ and $\widetilde{c}$ is defined by (4.5). We apply the definition of the sets $W_{1}, W_{1}^{c}$ and $W_{\epsilon 2}, W_{\epsilon 2}^{c}$ to write

$$
\begin{gathered}
D(u)=\left(\frac{1}{1-q u}\right)^{k_{1}^{2}+k_{2}^{2}} \prod_{\substack{j \in W_{1} \\
\epsilon_{j} \in\{ \pm 1\}}}\left(\frac{1}{1-q u e^{2 i \epsilon_{j} \theta_{j}}}\right)^{\frac{k_{j}\left(k_{j}+1\right)}{2}} \prod_{\substack{(1,2) \in W_{\epsilon 2} \\
\epsilon_{j} \in\{ \pm 1\}}}\left(\frac{1}{1-q u e^{i\left(\epsilon_{1} \theta_{1}+\epsilon_{2} \theta_{2}\right)}}\right)^{k_{1} k_{2}} \\
\times \prod_{\substack{j \in W_{1}^{c} \\
\epsilon_{j} \in\{ \pm 1\}}} \mathcal{Z}^{\frac{k_{j}\left(k_{j}+1\right)}{2}}\left(u e^{2 i \epsilon_{j} \theta_{j}}\right) \prod_{\substack{(1,2) \in W_{\epsilon 2}^{c} \\
\epsilon_{j} \in\{ \pm 1\}}} \mathcal{Z}^{k_{1} k_{2}}\left(u e^{i\left(\epsilon_{1} \theta_{1}+\epsilon_{2} \theta_{2}\right)}\right) C(u) \frac{(q u)^{-\left(k_{1}+k_{2}\right) X}}{u(1-q u)}
\end{gathered}
$$

\footnotetext{
${ }^{5}$ Note that for two dimensional correlations only one of the sets $W_{\epsilon 2}, W_{\epsilon 2}^{c}, \epsilon \in\{1,-1\}$ contains the "pair shift" $(1,2)$ but for higher dimensional correlations either of the sets $W_{\epsilon 2}, W_{\epsilon 2}^{c}$ may contain more than one "pair shift" which are of the form $\left(j_{1}, j_{2}\right)$.
} 


$$
\begin{gathered}
=\left(\frac{1}{1-q u}\right)^{k_{1}^{2}+k_{2}^{2}+1} \prod_{\substack{j \in W_{1} \\
\epsilon_{j} \in\{ \pm 1\}}}\left(\frac{1}{1-q u e^{2 i \epsilon_{j} \theta_{j}}}\right)^{\frac{k_{j}\left(k_{j}+1\right)}{2}} \prod_{\substack{(1,2) \in W_{\epsilon 2} \\
\epsilon_{j} \in\{ \pm 1\}}}\left(\frac{1}{1-q u e^{i\left(\epsilon_{1} \theta_{1}+\epsilon_{2} \theta_{2}\right)}}\right)^{k_{1} k_{2}} \\
\times(q u)^{-\left(k_{1}+k_{2}\right) X} \widetilde{E}(u),
\end{gathered}
$$

where

$$
\widetilde{E}(u)=\prod_{\substack{j \in W_{1}^{c} \\ \epsilon_{j} \in\{ \pm 1\}}} \mathcal{Z}^{\frac{k_{j}\left(k_{j}+1\right)}{2}}\left(u e^{2 i \epsilon_{j} \theta_{j}}\right) \prod_{\substack{(1,2) \in W_{\epsilon}^{c} \\ \epsilon, \epsilon_{1}, \epsilon_{2} \in\{ \pm 1\}}} \mathcal{Z}^{k_{1} k_{2}}\left(u e^{i\left(\epsilon_{1} \theta_{1}+\epsilon_{2} \theta_{2}\right)}\right) \frac{C(u)}{u}
$$

Note that $\widetilde{E}(u)$ is analytic on and inside the circle $\Gamma$ and it's radius of convergence is $\gg \frac{1}{g}$. Therefore for $\left|u-\frac{1}{q}\right|=O\left(\frac{1}{g}\right)$,

$$
\widetilde{E}(u)=\sum_{n=0}^{\infty} e_{n}(1-q u)^{n} .
$$

Next we evaluate the integral $\int_{\Gamma} D(u) d u$ which is equal to

$$
\int_{\Gamma} \frac{1}{(1-q u)^{V+1}}\left(1+\sum_{n=1}^{\infty} \frac{b_{n}}{(1-q u)^{n}}\right) \widetilde{E}(u)(q u)^{-\left(k_{1}+k_{2}\right) X} d u
$$

where

$$
\begin{aligned}
V=k_{1}^{2}+k_{2}^{2}+\sum_{j \in W_{1}} k_{j}\left(k_{j}+1\right)+\sum_{\substack{(1,2) \in W_{\epsilon 2} \\
\epsilon \in\{ \pm 1\}}} 2 k_{1} k_{2} \text { and } \\
1+\sum_{n=1}^{\infty} \frac{b_{n}}{(1-q u)^{n}}=\prod_{\substack{j \in W_{1} \\
\epsilon_{j} \in\{ \pm 1\}}}\left(1+\sum_{n=1}^{\infty}(-1)^{n}\left(\begin{array}{c}
\frac{k_{j}\left(k_{j}+1\right)}{2}+n \\
\frac{k_{j}\left(k_{j}+1\right)}{2}
\end{array}\right) \frac{\left(e^{-2 i \epsilon_{j} \theta_{j}}-1\right)^{n}}{(1-q u)^{n}}\right) \\
\times \prod_{\substack{(1,2) \in W_{\epsilon 2} \\
\epsilon, \epsilon_{1}, \epsilon_{2} \in\{ \pm 1\}}}\left(1+\sum_{m=1}^{\infty}(-1)^{m}\left(\begin{array}{c}
k_{1} k_{2}+m \\
k_{1} k_{2}
\end{array}\right) \frac{\left(e^{-i\left(\epsilon_{1} \theta_{1}+\epsilon_{2} \theta_{2}\right)}-1\right)^{m}}{(1-q u)^{m}}\right) .
\end{aligned}
$$

For $n \geq 0$, we deduce that

$$
\begin{aligned}
\int_{\Gamma} \frac{1}{(1-q u)^{V+1}} & \frac{b_{n}}{(1-q u)^{n}} \widetilde{E}(u)(q u)^{-\left(k_{1}+k_{2}\right) X} d u \\
& =e_{0} b_{n} \frac{F_{V+n}\left(\left(k_{1}+k_{2}\right) X\right)}{(V+n) !}+\sum_{l=1}^{V+n} e_{l} b_{n} \frac{F_{V+n-l}\left(\left(k_{1}+k_{2}\right) X\right)}{(V+n-l) !},
\end{aligned}
$$

where $F_{n}(x)=x(x+1)(x+2) \ldots(x+n-1)$, for $n \geq 1$ and $F_{0}(x)=1$.

From the choice of $X=\frac{g}{2\left(k_{1}+k_{2}\right)}$, right hand side of the above equation becomes

$$
\frac{e_{0} b_{n}}{(V+n) !}\left(\frac{g}{2}\right)^{V+n}+\sum_{l=1}^{V+n} \frac{e_{l} b_{n} d_{V+n-l}}{(V+n-l) !}\left(\frac{g}{2}\right)^{V+n-l}
$$


where

$$
d_{l}=1+\frac{l !}{e_{V+n-l}}\left(\frac{s_{l-1}^{(l)} e_{V+n-(l+1)}}{(l+1) !}+\frac{s_{l-1}^{(l+1)} e_{V+n-(l+2)}}{(l+2) !}+\ldots+\frac{s_{l-1}^{(V+n-2)} e_{1}}{(V+n-1) !}+\frac{s_{l-1}^{(V+n-1)} e_{0}}{(V+n) !}\right)
$$

with

$$
s_{k-i}^{(k)}=\sum_{1 \leq l_{1}<\ldots<l_{i} \leq k} l_{1} \ldots l_{i}, \quad i=1,2, \ldots, k .
$$

The coefficients $s_{k-i}^{(k)}$ are called the Stirling numbers of first kind and $s_{k-i}^{(k)} \leq(k+1)$ ! (see [16], equation (6.9)). For more details about $d_{l}$ see Appendix 7.2. Therefore, the integral (4.6) is equal to

$$
\begin{array}{r}
\frac{e_{0} g^{V}}{2^{V}} \sum_{n=0}^{\infty} \frac{b_{n} g^{n}}{2^{n}(V+n) !}+\sum_{l=1}^{V} \frac{e_{l} g^{V-l}}{2^{V-l}} \sum_{n=0}^{\infty} \frac{b_{n} d_{V+n-l} g^{n}}{2^{n}(V+n-l) !} \\
+\sum_{l=1}^{\infty} e_{V+l} \sum_{n=0}^{\infty} \frac{b_{n+l} d_{n} g^{n}}{2^{n} n !}
\end{array}
$$

We claim that the main contribution comes from only the first term of the above expression. To prove this, we have to find an upper bound for the coefficients $b_{n}, e_{l}$ and $d_{n}$. Let us denote

$$
M:=\max _{\substack{j \in W_{1} \\(1,2) \in W_{\epsilon 2} \\ \epsilon \in\{ \pm 1\}}}\left\{\frac{k_{j}\left(k_{j}+1\right)}{2}, k_{1} k_{2}\right\}, \quad \beta:=\max _{\substack{j \in W_{1} \\(1,2) \in W_{\epsilon 2} \\ \epsilon \in\{ \pm 1\}}}\left\{\left|1-e^{2 i \theta_{j}}\right|,\left|1-e^{i\left(\theta_{1} \pm \theta_{2}\right)}\right|\right\},
$$

and $2 w:=\max _{j}\left\{\left|W_{j}\right|\right\}$. We can write $b_{n}$ as

$$
\begin{aligned}
& b_{n}=(-1)^{n} \sum_{\substack{\sum n_{j}+m_{12}=n \\
n_{j}, m_{12} \geq 0 \\
j \in W_{1},(1,2) \in W_{\epsilon 2} \\
\epsilon \in\{ \pm 1\}}} \prod_{\substack{j \in W_{1} \\
\epsilon \in\{ \pm 1\}}}\left(\begin{array}{c}
\frac{k_{j}\left(k_{j}+1\right)}{2}+n_{j} \\
\frac{k_{j}\left(k_{j}+1\right)}{2}
\end{array}\right)\left(e^{-2 i \epsilon_{j} \theta_{j}}-1\right)^{n_{j}} \\
& \times \prod_{\substack{(1,2) \in W_{\epsilon 2} \\
\epsilon_{j} \in\{ \pm 1\}}}\left(\begin{array}{c}
k_{1} k_{2}+m_{12} \\
k_{1} k_{2}
\end{array}\right)\left(e^{-i\left(\epsilon_{1} \theta_{1}+\epsilon_{2} \theta_{2}\right)}-1\right)^{m_{12}} .
\end{aligned}
$$

Note that the number of terms such that $\sum n_{j}+m_{12}=n$ with $n_{j}, m_{12} \geq 0$ and $j \in$ $W_{1},(1,2) \in W_{\epsilon 2}$ is $\left(\begin{array}{c}w+n-1 \\ w-1\end{array}\right)$. Therefore, for large $g$ and $n \geq 1$, we obtain

$$
\left|b_{n}\right| \leq\left(\begin{array}{c}
w+n-1 \\
w-1
\end{array}\right)\left(\begin{array}{c}
M+n \\
M
\end{array}\right)^{w} \beta^{n} \leq a_{0} n^{t} \beta^{n}
$$

where $a_{0}, t$ are constants depend on $w$ and $M$.

Let $r$ be the radius of convergence of $\widetilde{E}(u)$. Note that $\frac{1}{g}=o(r)$. Hence $\lim _{n \rightarrow \infty} \frac{e_{n+1}}{e_{n}}=\frac{1}{r}=o(g)$, and this gives

$$
\left|e_{n}\right| \leq e_{0} a_{1}\left(\frac{2}{r}\right)^{n}
$$

where $a_{1} \in \mathbb{R}$ depends on $\widetilde{E}$. 
Note that,

$$
\begin{array}{r}
\frac{e_{l} d_{V+n-l}}{(V+n-l) !}=\frac{e_{l}}{(V+n-l) !}+\left(\frac{s_{V+n-l-1}^{(V+n-l)} e_{l-1}}{(V+n-l+1) !}+\frac{s_{V+n-l-1}^{(V+n-l+1)} e_{l-2}}{(V+n-l+2) !}+\ldots+\right. \\
\left.\frac{s_{V+n-l-1}^{(V+n-2)} e_{1}}{(V+n-1) !}+\frac{s_{V+n-l-1}^{(V+n-1)} e_{0}}{(V+n) !}\right),
\end{array}
$$

which implies together with (4.10),

$$
\frac{e_{l} d_{V+n-l}}{(V+n-l) !} \leq e_{l}+e_{l-1}+\ldots+e_{1}+e_{0} \leq e_{0} a_{1} \sum_{k=1}^{l}\left(\frac{2}{r}\right)^{k} \ll l\left(\frac{2}{r}\right)^{l} .
$$

By using the above bounds, the fact that $b_{0}=1$ and $\frac{1}{r}=o(g)$, the second sum of (4.8) is bounded by

$$
\begin{aligned}
& \ll_{\boldsymbol{k}} \sum_{l=1}^{V} l\left(\frac{2}{r}\right)^{l}\left(\frac{g}{2}\right)^{V-l}\left(1+\sum_{n=1}^{\infty} \frac{n^{t}(g \beta)^{n}}{2^{n}}\right) \\
& =o\left(g^{V} \prod_{j \in W_{1}^{c}} \frac{1}{\left|\theta_{j}\right|^{k_{j}\left(k_{j}+1\right)}} \prod_{\substack{(1,2) \in W_{\epsilon 2}^{c} \\
\epsilon \in\{ \pm 1\}}} \frac{1}{\left|\theta_{1}+\epsilon \theta_{2}\right|^{2 k_{1} k_{2}}}\right) .
\end{aligned}
$$

Since $|g \beta|<\widetilde{c}<1$ as $g \rightarrow \infty$, the inside $n$-sum in the above expression is $O(1)$. For any $l \geq 1$, using (4.9), we get

$$
\sum_{n=0}^{\infty} \frac{b_{n+l} d_{n} g^{n}}{2^{n} n !} \ll_{\boldsymbol{k}} l^{t} \beta^{l} \sum_{n=0}^{\infty} \frac{(n+l)^{t}}{l^{t}} \frac{(g \beta)^{n}}{2^{n}} \ll_{\boldsymbol{k}} l^{t} \beta^{l} \sum_{n=0}^{\infty} \frac{(n+1)^{t}(g \beta)^{n}}{2^{n}} \ll_{\boldsymbol{k}} l^{t} \beta^{l} .
$$

From the fact $\beta / r=o(1)$ and (4.10), the third sum of the equation (4.8) is bounded above by

$$
\begin{aligned}
& \ll_{k} \frac{1}{r^{V}} \sum_{l=1}^{\infty}\left(\frac{2 \beta}{r}\right)^{l} l^{t} \\
& =o\left(g^{V} \prod_{j \in W_{1}^{c}} \frac{1}{\left|\theta_{j}\right|^{k_{j}\left(k_{j}+1\right)}} \prod_{\substack{(1,2) \in W_{\epsilon 2}^{c} \\
\epsilon \in\{ \pm 1\}}} \frac{1}{\left|\theta_{1}+\epsilon \theta_{2}\right|^{2 k_{1} k_{2}}}\right) .
\end{aligned}
$$

Finally, we consider the first sum of the equation (4.8). Using the bound of $b_{n}$ (see (4.9)), we note that

$$
1 \ll \sum_{n=0}^{\infty} \frac{b_{n} g^{n}}{2^{n}(V+n) !} \ll \frac{1}{V !}+\sum_{n=1}^{\infty} \frac{n^{t}(\beta g)^{n}}{2^{n}(V+n) !}=O(1)
$$

where the implied constant depends on $\widetilde{c}$ and $\boldsymbol{k}(i . e ., V)$. Therefore, we conclude that

$$
\int_{\Gamma} D(u) d u \sim_{\boldsymbol{k}, \widetilde{c}} g^{V} \prod_{j \in W_{1}^{c}} \frac{1}{\left|\theta_{j}\right|^{k_{j}\left(k_{j}+1\right)}} \prod_{\substack{(1,2) \in W_{\epsilon 2}^{c} \\ \epsilon \in\{ \pm 1\}}} \frac{1}{\left|\theta_{1}+\epsilon \theta_{2}\right|^{2 k_{1} k_{2}}}
$$




$$
\sim_{\boldsymbol{k}, \widetilde{c}} g^{k_{1}^{2}+k_{2}^{2}} \prod_{j=1}^{2}\left(\min \left\{\frac{1}{\left|2 \theta_{j}\right|}, g\right\}\right)^{k_{j}\left(k_{j}+1\right)}\left(\min \left\{\frac{1}{\left|\theta_{1}-\theta_{2}\right|}, g\right\}\right)^{2 k_{1} k_{2}}\left(\min \left\{\frac{1}{\left|\theta_{1}+\theta_{2}\right|}, g\right\}\right)^{2 k_{1} k_{2}},
$$

as required.

Evaluation of infinite "single shift" and "pair shift". We claim that

$$
\sum_{\substack{j \in W_{1} \\ \epsilon_{j} \in\{ \pm 1\}}} \operatorname{Res}_{\substack{q e^{2 i \epsilon_{j} \theta_{j}}\\}} D(u)+\sum_{\substack{(1,2) \in W_{\epsilon 2} \\ \epsilon, \epsilon_{j} \in\{ \pm 1\}}} u=\frac{\operatorname{Res}_{q e^{i\left(\epsilon_{1} \theta_{1}+\epsilon_{2} \theta_{2}\right)}}}{} D(u)
$$

$=o\left(g^{k_{1}^{2}+k_{2}^{2}} \prod_{j=1}^{2}\left(\min \left\{\frac{1}{\left|2 \theta_{j}\right|}, g\right\}\right)^{k_{j}\left(k_{j}+1\right)}\left(\min \left\{\frac{1}{\left|\theta_{1}-\theta_{2}\right|}, g\right\}\right)^{2 k_{1} k_{2}}\left(\min \left\{\frac{1}{\left|\theta_{1}+\theta_{2}\right|}, g\right\}\right)^{2 k_{1} k_{2}}\right)$.

For the sake of simplicity, we will provide all the details of the proof of the claim (4.11) in the Appendix section.

4.2. Estimation of the sum $S_{1}$. We define

$$
\widetilde{\mathcal{L}}\left(u, \chi_{D}\right):=\mathcal{L}\left(\frac{v_{1}}{q^{1 / 2+\alpha_{1}}}, \chi_{D}\right)^{k_{1}} \mathcal{L}\left(\frac{v_{2}}{q^{1 / 2+\alpha_{2}}}, \chi_{D}\right)^{k_{2}}=\sum_{f \in \mathcal{M}} a_{f} \chi_{D}(f)\left(\frac{u}{\sqrt{q}}\right)^{d(f)},
$$

where $a_{f}$ is defined by (4.2). We begin with the integral

$$
I=\frac{1}{2 \pi i} \oint_{|u|=r} \widetilde{\mathcal{L}}\left(u, \chi_{D}\right) \frac{u^{-\left(k_{1}+k_{2}\right) X}}{(1-u)} \frac{d u}{u}, \quad r<1 .
$$

Integrating term by term to get

$$
I=\sum_{f \in \mathcal{M}_{\leq\left(k_{1}+k_{2}\right) X}} a_{f} \frac{\chi_{D}(f)}{|f|^{1 / 2}} .
$$

On the other hand we move the contour of integration to $|u|=q^{y}$, encountering a simple pole at $u=1, y>\frac{1}{2}$. In doing so, we obtain

$$
I=\widetilde{\mathcal{L}}\left(1, \chi_{D}\right)+\frac{1}{2 \pi i} \oint_{|u|=q^{y}} \widetilde{\mathcal{L}}\left(u, \chi_{D}\right) \frac{u^{-\left(k_{1}+k_{2}\right) X}}{(1-u)} \frac{d u}{u} .
$$

We use the Lindelöf bound $\widetilde{\mathcal{L}}\left(u, \chi_{D}\right) \ll q^{\varepsilon n}[[4]$, Theorem 3.3]6 to obtain

$$
\frac{1}{2 \pi i} \oint_{|u|=q^{y}} \widetilde{\mathcal{L}}\left(u, \chi_{D}\right) \frac{u^{-\left(k_{1}+k_{2}\right) X}}{(1-u)} \frac{d u}{u} \ll \frac{q^{\varepsilon n}}{q^{\left(\left(k_{1}+k_{2}\right) X+1\right) y}} .
$$

It follows that

$$
\widetilde{\mathcal{L}}\left(1, \chi_{D}\right)=\sum_{f \in \mathcal{M}_{\leq\left(k_{1}+k_{2}\right) X}} a_{f} \frac{\chi_{D}(f)}{|f|^{1 / 2}}+O_{\varepsilon}\left(\frac{q^{\varepsilon n}}{q^{\left(\left(k_{1}+k_{2}\right) X+1\right) y}}\right) .
$$

\footnotetext{
${ }^{6}$ One can use the Theorem 1.5 to get the better bound for the integral (4.12), but for our case Lindelöf bound is enough.
} 
From the approximation (4.13),

$$
\begin{aligned}
S_{1} & \gg\left|\sum_{D \in \mathcal{H}_{n}} \mathcal{L}\left(\frac{v_{1}}{q^{1 / 2+\alpha_{1}}}, \chi_{D}\right)^{k_{1}} \mathcal{L}\left(\frac{v_{2}}{q^{1 / 2+\alpha_{2}}}, \chi_{D}\right)^{k_{2}} \overline{\mathcal{L}_{\leq X}\left(\boldsymbol{v}, \chi_{D}\right)}\right| \\
& =\left|\sum_{f \in \mathcal{M}_{\leq\left(k_{1}+k_{2}\right) X}} \sum_{f^{\prime} \in \mathcal{M}_{\leq\left(k_{1}+k_{2}\right) X}} \frac{a_{f} \overline{a_{f^{\prime}}}}{\left|f f^{\prime}\right|^{1 / 2}} \sum_{D \in \mathcal{H}_{n}} \chi_{D}\left(f f^{\prime}\right)\right|+O_{\varepsilon}\left(q^{n \varepsilon}\left|\mathcal{H}_{n}\right| \frac{q^{(1 / 2+\varepsilon)\left(k_{1}+k_{2}\right) X}}{q^{\left(\left(k_{1}+k_{2}\right) X+1\right) y}}\right) \\
& =\left|S_{2}\right|+O_{\varepsilon}\left(q^{n \varepsilon}\left|\mathcal{H}_{n}\right| \frac{q^{(1 / 2+\varepsilon)\left(k_{1}+k_{2}\right) X}}{q^{\left(\left(k_{1}+k_{2}\right) X+1\right) y}}\right) .
\end{aligned}
$$

We choose $X=\frac{g}{2\left(k_{1}+k_{2}\right)}$ and $y=\frac{2}{3}$. Hence the estimate of $S_{2}$ gives us

$$
\begin{aligned}
S_{1} \gg_{\boldsymbol{k}}\left|\mathcal{H}_{n}\right| g^{k_{1}^{2}+k_{2}^{2}} \prod_{j=1}^{2} & \left(\min \left\{\frac{1}{\left|2 \theta_{j}\right|}, g\right\}\right)^{k_{j}\left(k_{j}+1\right)}\left(\min \left\{\frac{1}{\left|\theta_{1}-\theta_{2}\right|}, g\right\}\right)^{2 k_{1} k_{2}} \\
& \times\left(\min \left\{\frac{1}{\left|\theta_{1}+\theta_{2}\right|}, g\right\}\right)^{2 k_{1} k_{2}}+O\left(\left|\mathcal{H}_{n}\right| q^{-\frac{g}{12}+\varepsilon n}\right) .
\end{aligned}
$$

Inserting the estimates of $S_{1}$ and $S_{2}$ in (4.3) finishes the proof of Theorem 1.3 .

\section{Proof of Theorem 1.5}

To keep things simple we use the notation $\boldsymbol{v}$ instead of $\boldsymbol{v}^{(m)}$. The proof of the Theorem 1.5 will rely on getting an upper bound of the set

$$
\Upsilon_{n}(\boldsymbol{v}, V)=\#\left\{D \in \mathcal{H}_{n}: \sum_{j=1}^{m} 2 k_{j} \log \left|\mathcal{L}\left(\frac{v_{j}}{q^{\frac{1}{2}+\alpha_{j}}}, \chi_{D}\right)\right| \geq \mu(\boldsymbol{v}, g)+V\right\},
$$

for sufficiently large $n$ and for all $V>2$, where $\mu(\boldsymbol{v}, g)$ is defined by (1.5). Recall that

$$
2 g=n-1-\lambda \text {, }
$$

where $g$ and $\lambda$ are defined by (2.3) and (2.2) respectively. We can write

$$
\sum_{D \in \mathcal{H}_{n}}\left|\mathcal{L}\left(\frac{v_{1}}{q^{\frac{1}{2}+\alpha_{1}}}, \chi_{D}\right)\right|^{2 k_{1}} \ldots\left|\mathcal{L}\left(\frac{v_{m}}{q^{\frac{1}{2}+\alpha_{m}}}, \chi_{D}\right)\right|^{2 k_{m}}=\int_{-\infty}^{\infty} \Upsilon_{n}(\boldsymbol{v}, V) \exp (\mu(\boldsymbol{v}, g)+V) d V .
$$

We will estimate an upper bound of $\Upsilon_{n}(\boldsymbol{v}, V)$ for different ranges of $V$. The Lemma 3.4 lead us

$$
\begin{array}{r}
\sum_{j=1}^{m} 2 k_{j} \log \left|\mathcal{L}\left(\frac{v_{j}}{q^{\frac{1}{2}+\alpha_{j}}}, \chi_{D}\right)\right| \leq \frac{4 g}{N+1} \sum_{j=1}^{m} k_{j} \log \left(\frac{1+q^{-\alpha_{j}(N+1)}}{1+q^{-2(N+1)}}\right) \\
\quad+2 \Re \sum_{d(f) \leq N} \sum_{j=1}^{m} k_{j} \frac{a_{\alpha_{j}}(d(f)) \chi_{D}(f) \Lambda(f) v_{j}{ }^{d(f)}}{|f|^{\frac{1}{2}}}+O\left(\sum_{j=1}^{m} k_{j}\right) \\
\leq \frac{4 g K}{N+1} \log 2+2 \Re \sum_{d(f) \leq N} \sum_{j=1}^{m} k_{j} \frac{a_{\alpha_{j}}(d(f)) \chi_{D}(f) \Lambda(f) v_{j}{ }^{d(f)}}{|f|^{\frac{1}{2}}}+O(K),
\end{array}
$$


where

$$
K=\sum_{j=1}^{m} k_{j} \quad \text { and } \quad a_{\alpha_{j}}(d(f))=\frac{1}{d(f)|f|^{\alpha_{j}}}-\frac{1}{d(f)|f|^{2}}+O\left(\frac{1}{(N+1) q^{(N+1) \alpha_{j}}}\right) .
$$

Applying the prime polynomial theorem, the contribution from square polynomials $f=P^{2}$ to the second term of the right hand side of the above inequality is

$$
\begin{aligned}
& 2 \Re \sum_{d(P) \leq \frac{N}{2}} \sum_{j=1}^{m} k_{j} \frac{a_{\alpha_{j}}(2 d(P)) \chi_{D}(P) d(P) v_{j}^{2 d(P)}}{|P|}+O(\log \log n) \\
& \leq \mu(\boldsymbol{v}, g)+\frac{2 g K}{N+1}+O(\log \log n),
\end{aligned}
$$

where the error term $O(\log \log n)$ comes from the sum over $P$ such that $P \mid D$. Also it is easy to verify that the contribution from $f=P^{r}$ with $r \geq 3$ is $O(1)$. Therefore, we deduce that

$$
\sum_{j=1}^{m} 2 k_{j} \log \left|\mathcal{L}\left(\frac{v_{j}}{q^{\frac{1}{2}+\alpha_{j}}}, \chi_{D}\right)\right| \leq S_{1}(D)+S_{2}(D)+\mu(\boldsymbol{v}, g)+\frac{5 g K}{N+1}+O(\log \log n),
$$

where

$$
\begin{aligned}
& S_{1}(D)=2 \sum_{d(P) \leq N_{0}} \frac{\chi_{D}(P)}{|P|^{1 / 2}} \sum_{j=1}^{m} k_{j} a_{\alpha_{j}}(d(P)) d(P) \cos \left(\theta_{j} d(P)\right), \\
& S_{2}(D)=2 \sum_{N_{0}<d(P) \leq N} \frac{\chi_{D}(P)}{|P|^{1 / 2}} \sum_{j=1}^{m} k_{j} a_{\alpha_{j}}(d(P)) d(P) \cos \left(\theta_{j} d(P)\right) .
\end{aligned}
$$

We rewrite $\sigma(\boldsymbol{v}, g)$ as

$$
\sigma(\boldsymbol{v}, g)=2\left(\sum_{j=1}^{m} k_{j}^{2}\right) \log g+2 \sum_{j=1}^{m} k_{j}^{2} F_{j}+4 \sum_{i<j} k_{i} k_{j} F_{i, j}
$$

where

$$
F_{j}=\log \left(\min \left\{\frac{1}{2\left|\theta_{j}\right|}, g\right\}\right) \text { and } F_{i, j}=\log \left(\min \left\{\frac{1}{\left|\theta_{i}-\theta_{j}\right|}, g\right\}\right)+\log \left(\min \left\{\frac{1}{\left|\theta_{i}+\theta_{j}\right|}, g\right\}\right) .
$$

From now onward, for the sake of simplicity we write $\sigma(\boldsymbol{v}, g)$ simply as $\boldsymbol{\sigma}$. We consider various different range of $V$. The range $-\infty<V \leq \sqrt{\log g}$ yields

$$
\int_{-\infty}^{\infty} \Upsilon_{n}(\boldsymbol{v}, V) \exp (\mu(\boldsymbol{v}, g)+V) d V \ll\left|\mathcal{H}_{n}\right| \exp \left(\sqrt{\log g}+\mu(\boldsymbol{v}, g) \ll\left|\mathcal{H}_{n}\right| g^{o(1)} \exp (\mu(\boldsymbol{v}, g)) .\right.
$$

Applying Lemma 3.1, it is enough to assume that $\sqrt{\log g} \leq V \leq \frac{K g}{\log _{q} g}$. We define the quantity $A$ by

$$
A= \begin{cases}\frac{\log \boldsymbol{\sigma}}{2}, & \text { if } \sqrt{\log g} \leq V \leq \boldsymbol{\sigma} \\ \frac{\boldsymbol{\sigma} \log \boldsymbol{\sigma}}{2 V}, & \text { if } \boldsymbol{\sigma} \leq V \leq \frac{\boldsymbol{\sigma} \log \boldsymbol{\sigma}}{25 K} \\ 7 K, & \text { if } V>\frac{\boldsymbol{\sigma} \log \boldsymbol{\sigma}}{25 K}\end{cases}
$$


Let us consider

$$
\frac{g}{N+1}=\frac{V}{A} \quad \text { and } \quad N_{0}=\frac{N}{\log _{q} g} .
$$

Notice that, if $D \in \Upsilon_{n}(\boldsymbol{v}, V)$ then we must have either

$$
S_{1}(D) \geq V\left(1-\frac{6 K}{A}\right):=V_{1} \quad \text { or } \quad S_{2}(D) \geq \frac{K V}{A}:=V_{2} .
$$

To determine an upper bound of $\Upsilon_{n}(\boldsymbol{v}, V)$, we will actually examine the set

$$
\Upsilon_{n}\left(\boldsymbol{v}, V_{i}\right)=\#\left\{D \in \mathcal{H}_{n}: S_{i}(D) \geq V_{i}\right\}
$$

for $i=1,2$. We set $a_{j}(P):=a_{\alpha_{j}}(d(P)) d(P) \cos \left(\theta_{j} d(P)\right)$. So

$$
a_{j}(P)=\frac{\cos \left(\theta_{j} d(P)\right)}{|P|^{\alpha_{j}}}-\frac{\cos \left(\theta_{j} d(P)\right)}{|P|^{2}}+O\left(\frac{d(P)}{(N+1) q^{(N+1) \alpha_{j}}}\right) \ll 1 .
$$

Using Lemma 3.6, we obtain

$$
\begin{aligned}
\sum_{D \in \mathcal{H}_{n}}\left|S_{2}(D)\right|^{2 l} & \ll\left|\mathcal{H}_{n}\right| \frac{(2 l) !}{l ! 2^{l}}\left(\sum_{N_{0}<d(P) \leq N} \frac{\left(\sum_{j=1}^{m} 2 k_{j}\right)^{2}}{|P|}\right)^{l} \\
& \ll\left|\mathcal{H}_{n}\right| \frac{(2 l) !}{l ! 2^{l}}\left(4 K^{2}\left(\log \log _{q} g+O(1)\right)\right)^{l},
\end{aligned}
$$

for any $l$ such that $2 l N \leq n$, which implies that $l \leq \frac{g}{N}+\frac{1}{2 N} \leq \frac{2 V}{A}$.

Therefore, by using Markov's inequality and Stirling's formula, it follows that

$$
\begin{aligned}
\Upsilon_{n}\left(\boldsymbol{v}, V_{2}\right) & \leq V_{2}^{-2 l}\left(\sum_{D \in \mathcal{H}_{n}}\left|S_{2}(D)\right|^{2 l}\right) \\
& \ll\left|\mathcal{H}_{n}\right|\left(\frac{A}{K V}\right)^{2 l} \frac{(2 l) !}{l ! 2^{l}}\left(4 K^{2}\left(\log \log _{q} g+O(1)\right)\right)^{l} \\
& \ll\left|\mathcal{H}_{n}\right| \exp \left(-\frac{V}{2 A} \log V\right) .
\end{aligned}
$$

Again applying Lemma 3.6 and Stirling's formula, we get

$$
\begin{aligned}
\sum_{D \in \mathcal{H}_{n}}\left|S_{1}(D)\right|^{2 l} & \ll\left|\mathcal{H}_{n}\right| \frac{(2 l) !}{l ! 2^{l}}\left(\sum_{d(P) \leq N_{0}} \frac{1}{|P|}\left(\sum_{j=1}^{m} \frac{2 k_{j} \cos \left(\theta_{j} d(P)\right)}{\mid P \alpha_{j}}\right)^{2}\right)^{l} \\
& \ll\left|\mathcal{H}_{n}\right| \frac{(2 l) !}{l ! 2^{l}}\left(\sum _ { d ( P ) \leq N _ { 0 } } 4 \left(\sum_{j=1}^{m} \frac{k_{j}^{2} \cos ^{2}\left(\theta_{j} d(P)\right)}{|P|^{1+2 \alpha_{j}}}+2 \sum_{i<j} k_{i} k_{j} \frac{\cos \left(\theta_{i} d(P)\right) \cos \left(\theta_{j} d(P)\right)}{\left.\left.|P|^{1+\left(\alpha_{i}+\alpha_{j}\right)}\right)\right)^{l}}\right.\right. \\
& \ll\left|\mathcal{H}_{n}\right|\left(\frac{l \boldsymbol{\sigma}}{e}\right)^{l},
\end{aligned}
$$

for any $l$ such that $2 l N_{0} \leq n$, which implies that $l \leq \frac{V}{A} \log _{q} g$. Markov's inequality gives us

$$
\Upsilon_{n}\left(\boldsymbol{v}, V_{1}\right) \ll V_{1}^{-2 l}\left(\sum_{D \in \mathcal{H}_{n}}\left|S_{1}(D)\right|^{2 l}\right) \ll\left|\mathcal{H}_{n}\right|\left(\frac{l \boldsymbol{\sigma}}{e V_{1}^{2}}\right)^{l} .
$$

It is now convenient to consider the case when $V \leq \frac{\sigma^{2}}{K^{3}}$ and the case $V>\frac{\sigma^{2}}{K^{3}}$ separately. 
Case 1. Assume that $V \leq \frac{\sigma^{2}}{K^{3}}$. We choose $l=\left\lfloor\frac{V_{1}^{2}}{\sigma}\right\rfloor$. The definition of $A$ and this choice of $l$ implies that $l \leq \frac{V}{A} \log _{q} g$. In this case, we find that

$$
\Upsilon_{n}\left(\boldsymbol{v}, V_{1}\right) \ll\left|\mathcal{H}_{n}\right| \exp \left(l \log \left(\frac{l \boldsymbol{\sigma}}{e V_{1}^{2}}\right)\right) \ll\left|\mathcal{H}_{n}\right| \exp \left(-\frac{V_{1}^{2}}{\boldsymbol{\sigma}}\right) .
$$

Case 2. Assume that $V>\frac{\sigma^{2}}{K^{3}}$. We choose $l=\lfloor 10 V\rfloor$. Again from the definition of $A$, it is easy to see that this choice $l$ satisfies $l \leq \frac{V}{A} \log _{q} g$. Notice that $V>\frac{\sigma^{2}}{K^{3}}$, implies $\log V>2 \log \boldsymbol{\sigma}-3 \log K$. So, we have

$$
A=K \quad \text { and } \quad V_{1}^{2}=25 V^{2} .
$$

Hence, we conclude that

$$
\begin{aligned}
\Upsilon_{n}\left(\boldsymbol{v}, V_{1}\right) & \ll\left|\mathcal{H}_{n}\right| \exp \left(10 V \log \left(\frac{10 V \boldsymbol{\sigma}}{e V_{1}^{2}}\right)\right) \\
& \ll\left|\mathcal{H}_{n}\right| \exp (-4 V \log V),
\end{aligned}
$$

for sufficiently large $g$.

Therefore combining the above estimates, we deduce that

$$
\Upsilon_{n}(\boldsymbol{v}, V) \ll\left|\mathcal{H}_{n}\right|\left\{\exp \left(-\frac{V}{2 A} \log V\right)+\exp \left(-\frac{V_{1}^{2}}{\boldsymbol{\sigma}}\right)+\exp (-4 V \log V)\right\} .
$$

We extract the value of $V_{1}$ for various range of $V$ comes from the definition of $A$. If $\sqrt{\log g} \leq V \leq \boldsymbol{\sigma}$, then

$$
A=\frac{1}{2} \log \boldsymbol{\sigma} \quad \text { and } \quad V_{1}=V\left(1-\frac{12 K}{\log \boldsymbol{\sigma}}\right) .
$$

So, for sufficiently large $g$, (5.2) implies that

$$
\begin{aligned}
\Upsilon_{n}(\boldsymbol{v}, V) & \ll\left|\mathcal{H}_{n}\right| \exp \left(-\frac{V^{2}}{\sigma}\left(1-\frac{12 K}{\log \sigma}\right)^{2}\right) \\
& \ll\left|\mathcal{H}_{n}\right| \exp \left(-\frac{V^{2}}{\sigma}\left(1-\frac{24 K}{\log \sigma}\right)\right) .
\end{aligned}
$$

If $\boldsymbol{\sigma} \leq V \leq \frac{1}{25 K} \boldsymbol{\sigma} \log \boldsymbol{\sigma}$, then

$$
A=\frac{\boldsymbol{\sigma} \log \boldsymbol{\sigma}}{2 V} \quad \text { and } \quad V_{1}=V\left(1-\frac{12 K V}{\boldsymbol{\sigma} \log \boldsymbol{\sigma}}\right) .
$$

For this range of $V, \frac{\log V}{\sigma \log \sigma}>\frac{1}{\sigma}$ and hence from (5.2) we obtain

$$
\begin{aligned}
& \Upsilon_{n}(\boldsymbol{v}, V) \ll\left|\mathcal{H}_{n}\right|\{\exp (\left.-\frac{V^{2} \log V}{\boldsymbol{\sigma} \log \boldsymbol{\sigma}}\right)+\exp (-4 V \log V) \\
&\left.+\exp \left(-\frac{V^{2}}{\boldsymbol{\sigma}}\left(1-\frac{12 K V}{\boldsymbol{\sigma} \log \boldsymbol{\sigma}}\right)^{2}\right)\right\} \\
& \ll\left|\mathcal{H}_{n}\right| \exp \left(-\frac{V^{2}}{\boldsymbol{\sigma}}\left(1-\frac{24 K V}{\boldsymbol{\sigma} \log \boldsymbol{\sigma}}\right)\right) .
\end{aligned}
$$

Finally, if $V>\frac{1}{25 K} \boldsymbol{\sigma} \log \boldsymbol{\sigma}$, then

$$
A=7 K \quad \text { and } \quad V_{1}=\frac{V}{7} .
$$


So from (5.2), we get that

$$
\Upsilon_{n}(\boldsymbol{v}, V) \ll\left|\mathcal{H}_{n}\right| \exp \left(-\frac{V}{98 K} \log V\right) .
$$

Adding these estimates in (5.2) for different range of $V$, we conclude that

$$
\Upsilon_{n}(\boldsymbol{v}, V) \ll \begin{cases}\left|\mathcal{H}_{n}\right| n^{\varepsilon} \exp \left(-\frac{V^{2}}{\boldsymbol{\sigma}}\right) & , \text { if } 3 \leq V \leq 2021 \boldsymbol{\sigma}, \\ \left|\mathcal{H}_{n}\right| n^{\varepsilon} \exp (-4 V) & , \text { if } V>2021 \boldsymbol{\sigma} .\end{cases}
$$

Inserting (5.3) in (5.1) finishes the proof of Theorem 1.5,

\section{Proof of Theorem 1.6}

Let $C_{1 / g}$ be the circle in the complex plane whose center is origin and radius is $\frac{1}{g}$. By Cauchy's integral formula

$$
\mathcal{L}^{(l)}\left(q^{-1 / 2}, \chi_{D}\right)=\frac{l !}{2 \pi i} \oint_{C_{1 / g}} L\left(\frac{1}{2}+\theta, \chi_{D}\right) \frac{d \theta}{\theta^{l+1}}
$$

Notice that if $\theta=\alpha-\frac{i t}{\log q}$, then

$$
L\left(\frac{1}{2}+\theta, \chi_{D}\right)=\mathcal{L}\left(\frac{v}{q^{\alpha+1 / 2}}, \chi_{D}\right)
$$

where $\alpha=O\left(\frac{1}{g}\right)$. Therefore, applying Hölder's inequality, we see that

$$
\begin{aligned}
\sum_{D \in \mathcal{H}_{n}}\left|\mathcal{L}^{(l)}\left(q^{-1 / 2}, \chi_{D}\right)\right|^{k} & \leq\left(\frac{l !}{2 \pi}\right)^{k}\left(\sum_{D \in \mathcal{H}_{n}} \oint_{C_{1 / g}}\left|L\left(\frac{1}{2}+\theta, \chi_{D}\right)\right|^{k}|d \theta|\right)\left(\oint_{C_{1 / g}}|\theta|^{-\frac{k(l+1)}{(k-1)}}|d \theta|\right)^{(k-1)} \\
& \ll\left(\frac{l !}{2 \pi}\right)^{k}\left(\frac{2 \pi}{g}\right)^{k-1}\left(\frac{g}{2 \pi}\right)^{k(l+1)}\left(\sum_{D \in \mathcal{H}_{n}} \oint_{C_{1 / g}}\left|L\left(\frac{1}{2}+\theta, \chi_{D}\right)\right|^{k}|d \theta|\right) \\
& \ll\left(\frac{l !}{2 \pi}\right)^{k}\left(\frac{2 \pi}{g}\right)^{k}\left(\frac{g}{2 \pi}\right)^{k(l+1)} \max _{|\theta| \leq \frac{1}{g}} \sum_{D \in \mathcal{H}_{n}}\left|L\left(\frac{1}{2}+\theta, \chi_{D}\right)\right|^{k} .
\end{aligned}
$$

As a direct application of Theorem 1.5, we obtain

$$
\sum_{D \in \mathcal{H}_{n}}\left|\mathcal{L}\left(\frac{v}{q^{\alpha+1 / 2}}, \chi_{D}\right)\right|^{k} \ll_{\varepsilon}\left|\mathcal{H}_{n}\right| g^{\frac{k(k+1)}{2}+\varepsilon} .
$$

Using this upper bound to the above inequality, we conclude that

$$
\sum_{D \in \mathcal{H}_{n}}\left|\mathcal{L}^{(l)}\left(q^{-1 / 2}, \chi_{D}\right)\right|^{k} \ll_{\varepsilon}\left|\mathcal{H}_{n}\right| g^{\frac{k(k+1)}{2}+k l+\varepsilon} .
$$

Acknowledgements: We thank the anonymous referees for their valuable comments and insightful suggestions that have improved the quality of the manuscript. 


\section{REFERENCES}

[1] J. C. Andrade, Rudnick and Soundararajan's theorem for function fields, Finite Fields Appl., 37 (2016), 311-327.

[2] J. C. Andrade and J. P. Keating, The mean value of $L\left(\frac{1}{2}, \chi\right)$ in the hyperelliptic ensemble, J. Number Theory, 132 (2012), 2793-2816.

[3] J. C. Andrade, J. P. Keating, Conjectures for the integral moments and ratios of L-functions over function fields, J. Number Theory, 142 (2014), 102-148.

[4] S. A. Altuğ and J. Tsimerman, Metaplectic Ramanujan Conjecture Over Function Fields with Applications to Quadratic Forms, Int. Math. Res. Notices, 2014 (2013), 3465-3558.

[5] H. M. Bui, A. Florea, Zeros of quadratic Dirichlet L-functions in the hyperelliptic ensemble, Trans. Amer. Math. Soc., 370 (2018), 8013-8045.

[6] H. Bui and A. Florea, Hybrid Euler-Hadamard product for quadratic Dirichlet $L$-functions in function fields, Proc. Lond. Math. Soc., 117 (2018), 65-99.

[7] V. Chandee, On the correlation of shifted values of the Riemann zeta function, Q. J. Math., 62 (2011), 545-572.

[8] J. B. Conrey and D. W. Farmer, Mean values of L-functions and symmetry, Int. Math. Res. Notices, 17 (2000), 883-908.

[9] J. B. Conrey, D. W. Farmer, J. P. Keating, M. O. Rubinstein and N. C. Snaith, Integral moments of L-functions, Proc. London Math. Soc., 91 (2005), 33-104.

[10] J. B. Conrey, D. W. Farmer, J. P. Keating, M. O. Rubinstein and N. C. Snaith, Autocorrelation of random matrix polynomials, Commun. Math. Phys., 237 (2003), 365-395.

[11] J.B. Conrey, N.C. Snaith, Applications of the L-functions ratios conjectures, Proc. Lond. Math. Soc., 94 (2007), 594-646.

[12] A. Florea, Improving the error in the mean value of $L(1 / 2, \chi)$ in the hyperelliptic ensemble, Int. Math. Res. Notices, 20 (2017), 6119-6148.

[13] A. Florea, The second and third moment of $L(1 / 2, \chi)$ in the hyperelliptic ensemble, Forum Math., 29 (2017), 873-892.

[14] A. Florea, The fourth moment of quadratic Dirichlet L-functions over function fields, Geom. Funct. Anal., 27 (2017), 541-595.

[15] A. Granville and K. Soundararajan, Sieving and the Erdös-Kac theorem, Equidistribution in number theory, 237 (2007), 15-27.

[16] R. L. Graham, D. E. Knuth and O. Patashnik, Concrete Mathematics, Addison-Wesley, Reading, MA, 1989.

[17] A. Harper, Sharp conditional bounds for moments of the Riemann zeta function, preprint 2013, http://arxiv.org/abs/1305.4618.

[18] H. Jung, Note on the mean value of $L\left(\frac{1}{2}, \chi_{D}\right)$ in the hyperelliptic ensemble, J. Number Theory, 133 (2013), 2706-2714.

[19] J.P. Keating, B.E. Odgers, Symmetry transitions in random matrix theory and L-functions, Comm. Math. Phys., 281 (2008), 499-528.

[20] J. P. Keating and N. C. Snaith, Random Matrix Theory and $\zeta(1 / 2+i t)$, Commun. Math. Phys., 214 (2000), 57-89.

[21] J. P. Keating and N. C. Snaith, Random Matrix Theory and $L$-functions at $s=1 / 2$, Commun. Math. Phys., 214 (2000), 91-110.

[22] H.L. Montgomery, The pair correlation of zeros of the Riemann zeta-function, Proc. Symp. Pure Math., 24 (1973), 181-193.

[23] M. B. Milinovich, Upper bounds for moments of $\zeta^{\prime}(\rho)$, The Bulletin of the London Mathematical Society, 42 (2010), 28-44.

[24] M.B. Milinovich, Moments of the Riemann zeta-function at its relative extrema on the critical line, The Bulletin of the London Mathematical Society, 43 (2011), 1119-1129.

[25] M. Munsch, Shifted moments of $L$-functions and moments of theta functions, Mathematika, 63 (2017), 196-212.

[26] Micah B. Milinovich and Caroline L. Turnage-Butterbaugh. Moments of products of automorphic L-functions, J. Number Theory, 139 (2014), 175-204. 
[27] K Ramachandra, Some remarks on the mean value of the Riemann zeta-function and other Dirichlet series-II, Hardy-Ramanujan Journal, 3 (1980), 1-24.

[28] M. Rosen, Number theory in function fields, Graduate Texts in Mathematics, 210 (2002), SpringerVerlag, New York.

[29] Z. Rudnick, Traces of high powers of the Frobenius class in the hyperelliptic ensemble, Acta Arith, 143(1) (2010), 81-99.

[30] Z. Rudnick and K. Soundararajan, Lower bounds for moments of L-functions: Symplectic and orthogonal examples, pp. 293-303 in Multiple Dirichlet Series, Automorphic Forms, and Analytic Number Theory, edited by S. Friedberg et al., Proc. Sympos. Pure Math. 75, Amer. Math. Soc., Providence, RI, 2006.

[31] K. Sono, Lower bounds for the moments of the derivatives of the Riemann zeta-function and Dirichlet L-functions, Lith. Math. J., 52 (2012), 420-434.

[32] K. Sono, Upper bounds for the moments of derivatives of Dirichlet L-functions, Cent. Eur. J. Math., 12 (2014), 848-860.

[33] K. Soundararajan, Moments of the Riemann zeta-function, Annals of Mathematics, 170 (2009), 981-93.

[34] K. Soundararajan and M. young, The second moment of quadratic twists of modular $L$-functions, $J$. Eur. Math. Soc., 12 (2010), 1097-1116.

[35] A. Weil, Sur les courbes algébriques et les variétés qui s'en déduisent, Actualités Sci. Ind., 1041 (1948), Hermann et Cie., Paris.

\section{Appendix}

7.1. Proof of claim (4.11). We have to show that for $j \in W_{1}^{c}$,

$$
\begin{aligned}
& \operatorname{Res}_{u=\frac{1}{q e^{2 i \theta_{j}}}} D(u)= \\
& o\left(g^{k_{1}^{2}+k_{2}^{2}} \prod_{j=1}^{2}\left(\min \left\{\frac{1}{2\left|\theta_{j}\right|}, g\right\}\right)^{k_{j}\left(k_{j}+1\right)}\left(\min \left\{\frac{1}{\left|\theta_{1}-\theta_{2}\right|}, g\right\}\right)^{2 k_{1} k_{2}}\left(\min \left\{\frac{1}{\left|\theta_{1}+\theta_{2}\right|}, g\right\}\right)^{2 k_{1} k_{2}}\right),
\end{aligned}
$$

and for $(1,2) \in W_{2 \epsilon}^{c}, \epsilon \in\{ \pm 1\}$,

$$
\begin{gathered}
u=\frac{\operatorname{Res}}{q e^{i\left(\theta_{1}+\epsilon \theta_{2}\right)}} D(u)= \\
o\left(g^{k_{1}^{2}+k_{2}^{2}} \prod_{j=1}^{2}\left(\min \left\{\frac{1}{2\left|\theta_{j}\right|}, g\right\}\right)^{k_{j}\left(k_{j}+1\right)}\left(\min \left\{\frac{1}{\left|\theta_{1}-\theta_{2}\right|}, g\right\}\right)^{2 k_{1} k_{2}}\left(\min \left\{\frac{1}{\left|\theta_{1}+\theta_{2}\right|}, g\right\}\right)^{2 k_{1} k_{2}}\right) .
\end{gathered}
$$

We will prove the claim (7.1) and proof of the claim (7.2) follows in the similar way. We assume that $\epsilon, \epsilon_{j} \in\{1,-1\}$ for $j=1,2$. To prove the claim (7.1), without loss of generality, we assume that $1 \in W_{1}^{c}$, so $(1,2) \in W_{-2}^{c}$. Note that if $2 \in W_{1}^{c}$ and $(1,2) \in W_{2}^{c}$, then they are not closed to each other i.e., $\left|\theta_{1}-\theta_{2}\right| \gg \frac{1}{g},\left|\theta_{1}-\left(\theta_{1}-\theta_{2}\right)\right|=\left|\theta_{2}\right| \gg \frac{1}{g}$ and $\left|\theta_{2}-\left(\theta_{1}+\theta_{2}\right)\right|=\left|\theta_{1}\right| \gg \frac{1}{g}$, otherwise they will contained in the sets $W_{1}$ and $W_{2}$ respectively. By Cauchy's theorem, we obtain

$$
\underset{u=1 / q e^{2 i \theta_{j}}}{\operatorname{Res}} D(u)=\oint_{\widetilde{C}} D(u) d u, \quad j=1,2,
$$

\footnotetext{
${ }^{7}$ To estimate infinite pair shift for Theorem [1.5, one can follow the article of V. Chande [ [7, Appendix].
} 
where $\widetilde{C}$ is the circle centered at $u=1 / q e^{2 i \theta_{j}}$ with radius $\frac{\widetilde{c}}{g}$ and $\widetilde{c}$ is defined by (4.5). Note that, $\frac{1}{g}=o\left(\left|\theta_{j}\right|\right)$ and $\theta_{j}=o(1)$ for all $j$. For $u$ on the circle $\widetilde{C}$, we write

$$
\mathcal{Z}(u)=(1-q u)^{-1}=\left(1-e^{-2 i \theta_{1}}\right)^{-1}\left(1+\frac{e^{-2 i \theta_{1}}\left(1-q u e^{2 i \theta_{1}}\right)}{1-e^{-2 i \theta_{1}}}\right)^{-1} .
$$

Therefore, we get

$$
|\mathcal{Z}(u)| \ll \frac{1}{\left|\theta_{1}\right|} .
$$

If $2 \in W_{1}$, then it is easy to see that $\left|\theta_{1} \pm \theta_{2}\right| \sim\left|\theta_{1}\right|$. For $u$ on the circle $\widetilde{C}$,

$$
\mathcal{Z}\left(u e^{2 i \epsilon_{j} \theta_{2}}\right)=\left(1-q u e^{2 i \epsilon_{j} \theta_{2}}\right)^{-1}=\left(1-e^{-2 i \theta_{1}}\right)^{-1}\left(1+\frac{e^{-2 i \theta_{1}}\left(1-q u e^{2 i\left(\epsilon_{j} \theta_{2}-\theta_{1}\right)}\right)}{1-e^{-2 i \theta_{1}}}\right)^{-1},
$$

which implies that

$$
\left|\mathcal{Z}\left(u e^{2 i \epsilon_{j} \theta_{2}}\right)\right| \ll \frac{1}{\left|\theta_{1}\right|} .
$$

Also, if $(1,2) \in W_{2}$, then for $u$ on the circle $\widetilde{C}$, we see that

$$
\left|\mathcal{Z}\left(u e^{2 i\left(\epsilon_{1} \theta_{1}+\epsilon_{2} \theta_{2}\right)}\right)\right| \ll \frac{1}{\left|\theta_{1}\right|}
$$

For elements in the infinite single shift and pair shift, we have to partition the sets $W_{1}^{c}, W_{\epsilon 2}^{c}$ into three different subsets to estimate bounds for the corresponding zeta functions. For $2 \in W_{1}^{c}$, we divide the set $W_{1}^{c}$ into three subsets. First we define

$$
W_{11}^{c}:=\left\{2 \in W_{1}^{c}: \lim _{g \rightarrow \infty} \frac{\left|\theta_{1}\right|}{\left|\theta_{2}\right|}<+\infty \text { and } \lim _{g \rightarrow \infty} \frac{\theta_{1}}{\theta_{2}} \neq 1\right\} .
$$

If $2 \in W_{11}^{c}$, then for $u$ on the circle $\widetilde{C}$,

$$
\left|\mathcal{Z}\left(u e^{2 i \epsilon_{j} \theta_{2}}\right)\right| \ll \frac{1}{\left|\theta_{2}\right|} .
$$

Next, we consider

$$
W_{12}^{c}=\left\{2 \in W_{1}^{c}: \lim _{g \rightarrow \infty} \frac{\left|\theta_{1}\right|}{\left|\theta_{2}\right|}=\infty\right\} .
$$

For $2 \in W_{12}^{c}$ and $u$ on the circle $\widetilde{C}$, we obtain

$$
\left|\mathcal{Z}\left(u e^{2 i \epsilon_{j} \theta_{2}}\right)\right| \ll \frac{1}{\left|\theta_{1}\right|} .
$$

Lastly, let

$$
W_{13}^{c}=\left\{2 \in W_{1}^{c}: \lim _{g \rightarrow \infty} \frac{\theta_{1}}{\theta_{2}}=1\right\} .
$$

For $2 \in W_{13}^{c}$ and $u$ on the circle $\widetilde{C}$,

$$
\left|\mathcal{Z}\left(u e^{2 i \epsilon_{j} \theta_{2}}\right)\right| \ll \frac{1}{\left|\theta_{1}-\theta_{2}\right|} .
$$

Similarly, for $(1,2) \in W_{\epsilon 2}^{c}$, we define

$$
{ }^{1} W_{\epsilon 2}^{c}=\left\{(1,2) \in W_{\epsilon 2}^{c}: \lim _{g \rightarrow \infty} \frac{\left|\theta_{1}\right|}{\left|\theta_{1}-\epsilon \theta_{2}\right|}<+\infty \text { and } \lim _{g \rightarrow \infty} \frac{\theta_{1}}{\left(\theta_{1}-\epsilon \theta_{2}\right)} \neq 1\right\} .
$$


In this case, for $u$ on the circle $\widetilde{C}$,

$$
\left|\mathcal{Z}\left(u e^{i\left(\theta_{1}-\epsilon \theta_{2}\right)}\right)\right| \ll \frac{1}{\left|\theta_{1}-\theta_{2}\right|} .
$$

Let

$$
{ }^{2} W_{\epsilon 2}^{c}=\left\{(1,2) \in W_{\epsilon 2}^{c}: \lim _{g \rightarrow \infty} \frac{\left|\theta_{1}\right|}{\left|\theta_{1}-\epsilon \theta_{2}\right|}=+\infty\right\} .
$$

Inside the set ${ }^{2} W_{\epsilon 2}^{c}$, for $u$ on the circle $\widetilde{C}$,

$$
\left|\mathcal{Z}\left(u e^{i\left(\theta_{1}-\epsilon \theta_{2}\right)}\right)\right| \ll \frac{1}{\left|\theta_{1}\right|} .
$$

Lastly, we consider

$$
{ }^{3} W_{\epsilon 2}^{c}=\left\{(1,2) \in W_{\epsilon 2}^{c}: \lim _{g \rightarrow \infty} \frac{\theta_{1}}{\left(\theta_{1}-\epsilon \theta_{2}\right)}=1\right\} .
$$

For $u$ on the circle $\widetilde{C}$,

$$
\left|\mathcal{Z}\left(u e^{i\left(\theta_{1}-\epsilon \theta_{2}\right)}\right)\right| \ll \frac{1}{\left|\theta_{2}\right|} .
$$

Using these bounds for the zeta functions, we conclude that

$$
\begin{aligned}
\oint_{\widetilde{C}} D(u) d u \ll & g^{\frac{k_{1}\left(k_{1}+1\right)}{2}-1}\left|\theta_{1}\right|^{-\left(k_{1}^{2}+k_{2}^{2}+\frac{k_{1}\left(k_{1}+1\right)}{2}\right)} \min \left\{\frac{1}{\left|\theta_{1}\right|}, \frac{1}{\left|\theta_{2}\right|}, \frac{1}{\left|\theta_{1}-\theta_{2}\right|}\right\}^{k_{2}\left(k_{2}+1\right)} \\
& \times \min \left\{\frac{1}{\left|\theta_{1}\right|}, \frac{1}{\left|\theta_{2}\right|}, \frac{1}{\left|\theta_{1}-\theta_{2}\right|}\right\}^{2 k_{1} k_{2}} \min \left\{\frac{1}{\left|\theta_{1}\right|}, \frac{1}{\left|\theta_{2}\right|}, \frac{1}{\left|\theta_{1}+\theta_{2}\right|}\right\}^{2 k_{1} k_{2}}
\end{aligned}
$$

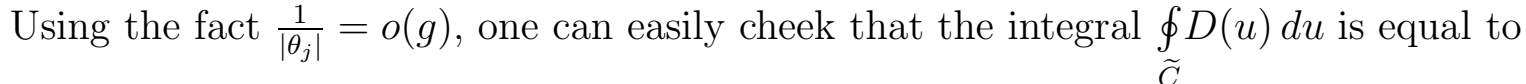

$o\left(g^{k_{1}^{2}+k_{2}^{2}} \prod_{j=1}^{2}\left(\min \left\{\frac{1}{\left|2 \theta_{j}\right|}, g\right\}\right)^{k_{j}\left(k_{j}+1\right)}\left(\min \left\{\frac{1}{\left|\theta_{1}-\theta_{2}\right|}, g\right\}\right)^{2 k_{1} k_{2}}\left(\min \left\{\frac{1}{\left|\theta_{1}+\theta_{2}\right|}, g\right\}\right)^{2 k_{1} k_{2}}\right)$,

and we obtain the claim (7.1).

7.2. Deduction of $d_{n}$. We start with the expression (4.7), i.e.,

$$
e_{0} b_{n} \frac{F_{V+n}\left(\left(k_{1}+k_{2}\right) X\right)}{(V+n) !}+\sum_{l=1}^{V+n} e_{l} b_{n} \frac{F_{V+n-l}\left(\left(k_{1}+k_{2}\right) X\right)}{(V+n-l) !}
$$

where $F_{n}(x)=x(x+1)(x+2) \ldots(x+n-1)$, for $n \geq 2$ and $F_{0}(x)=1, F_{1}(x)=x$. We expand $F_{n}(x)$ to get

$$
F_{n}(x)=x\left(x^{n-1}+s_{n-2}^{(n-1)} x^{n-2}+s_{n-3}^{(n-1)} x^{n-3}+s_{n-4}^{(n-1)} x^{n-4}+\ldots+s_{0}^{(n-1)}\right)
$$

with

This gives us

$$
s_{k-i}^{(k)}=\sum_{1 \leq l_{1}<\ldots<l_{i} \leq k} l_{1} \ldots l_{i}, \quad i=1,2, \ldots, k .
$$

$$
e_{0} b_{n} \frac{F_{V+n}(x)}{(V+n) !}+e_{1} b_{n} \frac{F_{V+n-1}(x)}{(V+n-1) !}+e_{2} b_{n} \frac{F_{V+n-2}(x)}{(V+n-2) !}+e_{3} b_{n} \frac{F_{V+n-3}(x)}{(V+n-3) !}
$$




$$
\begin{gathered}
\quad+\ldots+e_{V+n-1} b_{n} F_{1}(x)+e_{V+n} b_{n} F_{0}(x) \\
=\frac{e_{0} b_{n}}{(V+n) !}\left(x^{V+n}+s_{V+n-2}^{(V+n-1)} x^{V+n-1}+s_{V+n-3}^{(V+n-1)} x^{V+n-2}+\ldots+s_{0}^{(V+n-1)} x\right) \\
+\frac{e_{1} b_{n}}{(V+n-1) !}\left(x^{V+n-1}+s_{V+n-3}^{(V+n-2)} x^{V+n-2}+s_{V+n-4}^{(V+n-2)} x^{V+n-3}+\ldots+s_{0}^{(V+n-2)} x\right) \\
+\frac{e_{2} b_{n}}{(V+n-2) !}\left(x^{V+n-2}+s_{V+n-4}^{(V+n-3)} x^{V+n-3}+s_{V+n-5}^{(V+n-3)} x^{V+n-4}+\ldots+s_{0}^{(V+n-3)} x\right) \\
+\frac{e_{V+n-3} b_{n}}{3 !}\left(x^{3}+s_{1}^{(2)} x^{2}+s_{0}^{(2)} x\right)+\frac{e_{V+n-2} b_{n}}{2 !}\left(x^{2}+s_{0}^{(1)} x\right)+e_{V+n-1} b_{n} x+e_{V+n} b_{n} . \\
\quad=\frac{e_{0} b_{n}}{(V+n) !} x^{V+n}+\frac{e_{1} b_{n} d_{V+n-1}}{(V+n-1) !} x^{V+n-1}+\frac{e_{2} b_{n} d_{V+n-2}}{(V+n-2) !} x^{V+n-2}+\frac{e_{3} b_{n} d_{V+n-3}}{(V+n-3) !} x^{V+n-3}+\ldots+\frac{e_{V+n-3} b_{n} d_{3}}{3 !} x^{3}+\frac{e_{V+n-2} b_{n} d_{2}}{2 !} x^{2}+e_{V+n-1} b_{n} d_{1} x+e_{V+n} b_{n} d_{0},
\end{gathered}
$$

where $d_{0}=1$, and $1 \leq l \leq V+n-1$,

$$
d_{l}=1+\frac{l !}{e_{V+n-l}}\left(\frac{s_{l-1}^{(l)} e_{V+n-(l+1)}}{(l+1) !}+\frac{s_{l-1}^{(l+1)} e_{V+n-(l+2)}}{(l+2) !}+\ldots+\frac{s_{l-1}^{(V+n-2)} e_{1}}{(V+n-1) !}+\frac{s_{l-1}^{(V+n-1)} e_{0}}{(V+n) !}\right) .
$$

Indian Statistical Institute, Kolkata, West Bengal 700108, India

Email address, Pranendu Darbar: darbarpranendu100@gmail.com

Indian Statistical Institute, Kolkata, West Bengal 700108, India

Email address, Gopal Maiti: g.gopaltamluk@gmail.com 